\title{
Caprazamycins, Novel Lipo-nucleoside Antibiotics, from Streptomyces sp.
}

\author{
II. Structure Elucidation of Caprazamycins \\ Masayuki Igarashi, Yoshiaki Takahashi, Tetsuo Shitara, Hikaru Nakamura, \\ Hiroshi Naganawa, Toshiaki Miyake, Yuzuru Akamatsu
}

Received: February 15, 2005 / Accepted: April 28, 2005

(C) Japan Antibiotics Research Association

\begin{abstract}
Novel antibiotics, active against acid-fast bacteria, caprazamycins, were isolated from the culture broth of Streptomyces sp. MK730-62F2. The planar structures of the compounds were determined by 2D NMR spectroscopic study. Furthermore, the absolute structure of caprazamycin B (2) was established by NMR spectroscopy and X-ray crystallography of its degradation products and by total synthesis of the 5-amino-5-deoxy-D-ribose moiety. In the course of degradation studies of $\mathbf{2}$ under alkaline and acidic conditions, we obtained the two core components, caprazene (11) and caprazol (14), respectively, in high yield.
\end{abstract}

Structurally, caprazamycins belong to a family of lipouridyl antibiotics, which have been discovered as specific inhibitors of a bacterial translocase.

Keywords caprazamycin, antituberculous antibiotics, absolute structure, caprazol, caprazen

\section{Introduction}

Caprazamycins (CPZs, Fig. 1) are novel lipo-nucleoside antibiotics, produced by Streptomyces sp. MK730-62F2.
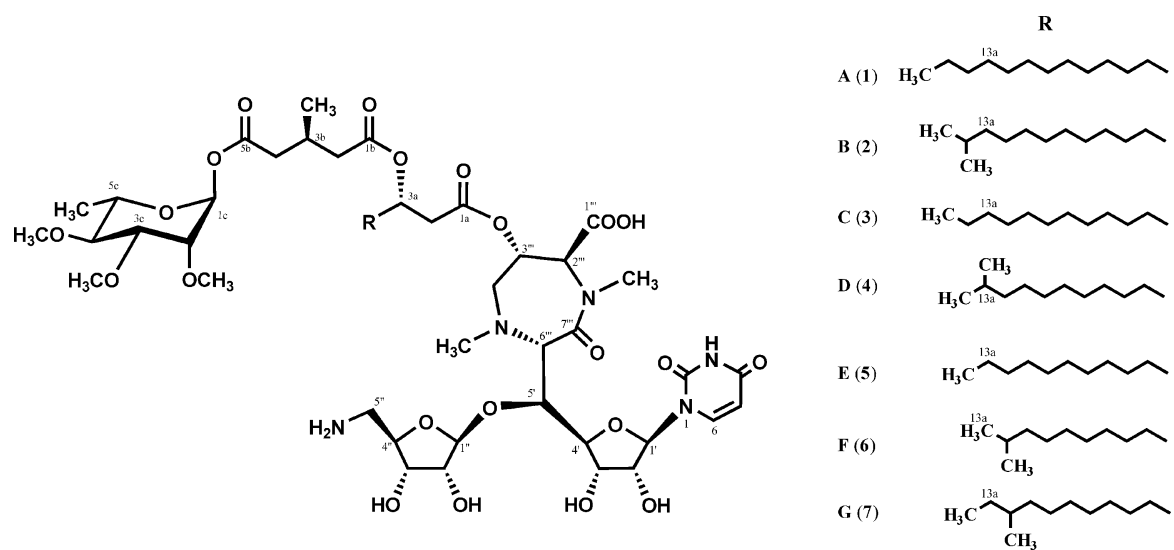

Fig. 1 Structures of caprazamycins.

M. Igarashi (Corresponding author), T. Shitara, H. Nakamura, H. Naganawa, Y. Akamatsu: Microbial Chemistry Research Center, 3-14-23 Kamiosaki, Shinagawa-ku, Tokyo 141-0021, Japan, E-mail: igarashim@bikaken.or.jp
Y. Takahashi, T. Miyake: Hiyoshi Medicinal Chemistry Research Institute, Microbial Chemistry Research Center, 3-3417, Ida, Nakahara-ku, Kawasaki-shi, Kanagawa, 211-0035, Japan 
They show activity against acid-fast bacteria including Mycobacterium tuberculosis and M. avium complex (MAC). A taxonomic study and fermentation of the producing strain together with the isolation and biological activities of CPZs have been reported in the preceding papers [1 3].

In this paper, we report the physico-chemical properties, degradation studies and structural elucidation of CPZs. Anti-M. tuberculosis activities, anti-MAC activities and therapeutic efficacy of the compounds in a murine pulmonary tuberculosis model will be described in a separate paper [4].

\section{Results and Discussion}

\section{Structure Determination}

The physico-chemical properties of CPZ-A, B, C, D, E, F and $G(\mathbf{1}, \mathbf{2}, \mathbf{3}, \mathbf{4}, \mathbf{5}, \mathbf{6}$ and 7) are summarized in Table 1. The molecular formulae of $\mathbf{1}, \mathbf{2}, \mathbf{3}, \mathbf{4}, \mathbf{5}, \mathbf{6}$ and 7 were established as $\mathrm{C}_{53} \mathrm{H}_{87} \mathrm{~N}_{5} \mathrm{O}_{22}, \mathrm{C}_{53} \mathrm{H}_{87} \mathrm{~N}_{5} \mathrm{O}_{22}, \mathrm{C}_{52} \mathrm{H}_{85} \mathrm{~N}_{5} \mathrm{O}_{22}$, $\mathrm{C}_{52} \mathrm{H}_{85} \mathrm{~N}_{5} \mathrm{O}_{22}, \mathrm{C}_{51} \mathrm{H}_{83} \mathrm{~N}_{5} \mathrm{O}_{22}, \mathrm{C}_{51} \mathrm{H}_{83} \mathrm{~N}_{5} \mathrm{O}_{22}$ and $\mathrm{C}_{52} \mathrm{H}_{85} \mathrm{~N}_{5} \mathrm{O}_{22}$, respectively, on the basis of HR-FAB-MS and NMR spectral analyses. The characteristic UV absorptions at 261 262 nm in methanol and the NMR spectra of CPZs suggested the presence of uridyl moiety in the molecules. The ${ }^{13} \mathrm{C}$ NMR data of CPZs are shown in Table 2. The multiplicity of carbon signals was determined by DEPT experiments. The ${ }^{1} \mathrm{H}$ and ${ }^{13} \mathrm{C}$ NMR spectra of CPZs showed similar signal patterns, except for those of the acylated side chain moiety. The DEPT and HMQC (heteronuclear multiple quantum coherence) spectra of 2 revealed the presence of nine methyl, fourteen methylene, twenty two methine groups and seven carbonyl carbons. Of the twenty two methine groups, two were presumed to be olefinic and three anomeric. The ${ }^{1} \mathrm{H}$ and ${ }^{13} \mathrm{C}$ NMR data (Table 3) of $\mathbf{2}$ were similar to those of liposidomycins [5] except for signals assigned to the sugar portion.

The ${ }^{1} \mathrm{H}-{ }^{1} \mathrm{H}$ COSY and HMBC (hetero nuclear multiple bond correlation) spectra suggested that 2 contained five partial structures (a, b, c, d and e) and two $\mathrm{N}-\mathrm{CH}_{3}$ groups as shown in Fig. 2. The ${ }^{13} \mathrm{C}-{ }^{1} \mathrm{H}$ couplings of ${ }^{2} J$ and ${ }^{3} J$ observed in the HMBC experiments gave the following evidence. The cross peaks from $\delta 8.07(6-\mathrm{H})$ to $\delta 150.7$ (C2), $\delta 163.9(\mathrm{C}-4)$ and $\delta 90.2\left(\mathrm{C}-1^{\prime}\right)$, from $\delta 4.15\left(6^{\prime \prime \prime}-\mathrm{H}\right)$ to $\delta 170.8\left(\mathrm{C}-7^{\prime \prime \prime}\right)$ supported partial structure a. The cross peak from $\delta 5.62\left(1^{\prime \prime}-\mathrm{H}\right)$ to $\delta 79.1\left(\mathrm{C}-4^{\prime \prime}\right)$ supported partial structure b. The cross peak from $\delta 4.94\left(2^{\prime \prime \prime}-\mathrm{H}\right)$ to $\delta 170.8$ $\left(\mathrm{C}-1^{\prime \prime \prime}\right)$ supported partial structure $\mathbf{c}$. The cross peaks from $\delta 5.48(3 \mathrm{a}-\mathrm{H})$ to $\delta 169.7(\mathrm{C}-1 \mathrm{a})$ and $\delta 171.7(\mathrm{C}-1 \mathrm{~b})$, from $\delta$ $2.62(2 \mathrm{~b}-\mathrm{H})$ to $\delta 171.7(\mathrm{C}-1 \mathrm{~b})$, and from $\delta 2.64$ and 2.47
(4b-H) to $\delta 171.0(\mathrm{C}-5 \mathrm{~b})$ supported partial structure $\mathbf{d}$. Moreover, the cross peaks from $\delta 6.34(1 \mathrm{c}-\mathrm{H})$ to $\delta 70.2(\mathrm{C}$ $5 \mathrm{c})$, from $\delta 3.50\left(2 \mathrm{c}-\mathrm{OCH}_{3}\right)$ to $\delta 76.0(\mathrm{C}-2 \mathrm{c})$, from $\delta 3.53$ $\left(3 \mathrm{c}-\mathrm{OCH}_{3}\right)$ to $\delta 80.9(\mathrm{C}-3 \mathrm{c})$ and from $\delta 3.56\left(4 \mathrm{c}-\mathrm{OCH}_{3}\right)$ to $\delta 81.6(\mathrm{C}-4 \mathrm{c})$ supported partial structure e.

The connections between the five partial structures $(\mathbf{a}, \mathbf{b}$, c, $\mathbf{d}$ and e) and two $\mathrm{N}-\mathrm{CH}_{3}$ groups were revealed by the HMBC spectrum as shown in Fig. 3. The anomeric proton at $\delta 5.62\left(1^{\prime \prime}-\mathrm{H}\right)$ was coupled with the methine carbon bearing an oxgen atom at $\delta 76.2\left(\mathrm{C}-5^{\prime}\right)$. The methine proton bearing an oxgen atom at $\delta 5.75\left(3^{\prime \prime \prime}-\mathrm{H}\right)$ was coupled with the carbonyl carbon at $\delta 169.7$ (C-1a). The anomeric proton at $\delta 6.34(1 \mathrm{c}-\mathrm{H})$ of the sugar moiety e was coupled with the carbonyl carbon at $\delta 171.0(\mathrm{C}-5 \mathrm{~b})$. The $N$ methyl protons at $\delta 2.72\left(5^{\prime \prime \prime}-\mathrm{NCH}_{3}\right)$ were coupled with the methine carbon at $\delta 63.8\left(\mathrm{C}-6^{\prime \prime \prime}\right)$ and the methylene carbon at $\delta 57.1\left(\mathrm{C}-4^{\prime \prime \prime}\right)$. Moreover, the $N$-methyl protons at $\delta 3.35$ $\left(8^{\prime \prime \prime}-\mathrm{NCH}_{3}\right)$ were coupled with the methine carbon at $\delta 63.9$ $\left(\mathrm{C}-2^{\prime \prime \prime}\right)$ and the carbonyl carbon at $\delta 170.8\left(\mathrm{C}-7^{\prime \prime \prime}\right)$. Based on the above observations, the planar structure of caprazamycin B (2) was elucidated as shown in Fig. 3.

\section{Stereochemistry of Caprazamycin B}

The absolute structure of $\mathbf{2}$ was determined by NMR spectroscopy and X-ray crystallography of its degradation products, including the two core components.

Acid hydrolysis of $\mathbf{2}$ with $80 \%$ trifluoroacetic acid in methanol at room temperature gave the aglycone, designated as caprazamycin B1 (8) and 2,3,4-tri-O-methylL-rhamnose (9) in high yield, respectively (Scheme 1). To ascertain the L-rhamnose structure of $\mathbf{9}$, the sugar was converted to the known methyl glycosides. Treatment of 9 with acidic methanol at $50^{\circ} \mathrm{C}$ gave a mixture of the $\quad \alpha$-glycoside, methyl 2,3,4-tri- $O$-methyl- $\alpha$-Lrhamnopyranoside (10a), and its $\beta$-anomer 10b. Isolation of the anomeric mixture by column chromatography gave 10a as a syrup, of which the physico-chemical properties were identical with those of the authentic sample [6 8]. The glycosidic linkage of L-rhamnose to caprazamycin B (2) was deduced from the ${ }^{13} \mathrm{C}-\mathrm{NMR}$ spectra. The ${ }^{1} J_{\mathrm{C}-\mathrm{H}}$ coupling constant $(167 \mathrm{~Hz})$ of the anomeric carbon of $\alpha$ glycoside 10a was larger than those of the $\beta$-anomer 10b $\left({ }^{1} J_{\mathrm{C}-\mathrm{H}}=153 \mathrm{~Hz}\right)$. On the other hand, the anomeric carbon of 2,3,4-tri- $O$-methylated rhamnose in $\mathbf{2}$ showed a larger value $\left({ }^{1} J_{\mathrm{Clc}-\mathrm{H} 1 \mathrm{c}}=174 \mathrm{~Hz}\right.$, in dimethylsulfoxide- $\left.d_{6}\right)$. Because it is known that introduction of an acyl group instead of a methoxyl group at an anomeric position increases the ${ }^{1} J_{\mathrm{C}-\mathrm{H}}$ values to some extent [9], the mode of sugar linkage in $\mathbf{2}$ is regarded as $\alpha$.

Further hydrolysis of $\mathbf{8}$ with $80 \%$ aqueous acetic acid at $70^{\circ} \mathrm{C}$ gave the unsaturated compound $\mathbf{1 1}$, designated as 
Table 1 Physico-chemical properties of CPZs

\begin{tabular}{|c|c|c|c|c|}
\hline & Caprazamycin A (1) & Caprazamycin B (2) & Caprazamycin C (3) & Caprazamycin D (4) \\
\hline Appearance & colorless powder & colorless powder & colorless powder & colorless powder \\
\hline Molecular formula & $\begin{array}{l}\mathrm{C}_{53} \mathrm{H}_{87} \mathrm{~N}_{5} \mathrm{O}_{22} \\
\text { [obsd. } 1146.5933 \\
\quad(\mathrm{M}+\mathrm{H})^{+}, \text {error }+1.2 \text { ] }\end{array}$ & $\begin{array}{l}\mathrm{C}_{53} \mathrm{H}_{87} \mathrm{~N}_{5} \mathrm{O}_{22} \\
\text { [obsd. } 1144.5750 \\
\quad(\mathrm{M}-\mathrm{H})^{-} \text {, error }-1.4 \text { ] }\end{array}$ & $\begin{array}{l}\mathrm{C}_{52} \mathrm{H}_{85} \mathrm{~N}_{5} \mathrm{O}_{22} \\
\text { [obsd. } 1132.5747 \\
\quad(\mathrm{M}+\mathrm{H})^{+}, \text {error }-1.7 \text { ] }\end{array}$ & $\begin{array}{l}\mathrm{C}_{52} \mathrm{H}_{85} \mathrm{~N}_{5} \mathrm{O}_{22} \\
\text { [obsd. } 1132.5747 \\
\quad(\mathrm{M}+\mathrm{H})^{+}, \text {error }-1.7 \text { ] }\end{array}$ \\
\hline \multirow[t]{2}{*}{ FAB-MS $(m / z)$} & $1146(\mathrm{M}+\mathrm{H})^{+}$ & $1146(\mathrm{M}+\mathrm{H})^{+}$ & $1132(\mathrm{M}+\mathrm{H})^{+}$ & $1132(\mathrm{M}+\mathrm{H})^{+}$ \\
\hline & $1144(\mathrm{M}-\mathrm{H})^{-}$ & $1144(\mathrm{M}-\mathrm{H})^{-}$ & $1130(\mathrm{M}-\mathrm{H})^{-}$ & $1130(\mathrm{M}-\mathrm{H})^{-}$ \\
\hline$[\alpha]_{D}^{23}$ & -1.4 (с 0.83, DMSO) & -2.6 (с 0.91, DMSO) & -1.1 (с 1.33, DMSO) & -3.0 (c 1, MeOH) \\
\hline$U V \lambda_{\max }^{\mathrm{MeOH}} \mathrm{nm}(\varepsilon)$ & $261(7,400)$ & $262(8,000)$ & $261(8,300)$ & $262(9,200)$ \\
\hline $0.03 \mathrm{M} \mathrm{HCl}-\mathrm{MeOH}$ & $260(7,200)$ & $262(7,800)$ & $261(8,200)$ & $261(8,900)$ \\
\hline $0.03 \mathrm{M} \mathrm{NaOH}-\mathrm{MeOH}$ & $261(5,600)$ & $262(6,400)$ & $260(6,400)$ & $260(7,300)$ \\
\hline $\mathrm{IR} v_{\max }(\mathrm{KBr}) \mathrm{cm}^{-1}$ & $\begin{array}{l}3421,2925,2854,1740, \\
1697 \text { (sh), 1675, } \\
1635 \text { (sh), 1269, 1466, } \\
1387,1268,1124,1103\end{array}$ & $\begin{array}{l}3400,2925,2854,1739 \\
1701 \text { (sh), 1674, } \\
1635 \text { (sh),1467, 1386, } \\
1193,1126,1001\end{array}$ & $\begin{array}{l}3421,2925,2854,1739 \\
1697 \text { (sh), 1675, } \\
1637 \text { (sh), 1465, 1386, } \\
1268,1124,1103\end{array}$ & 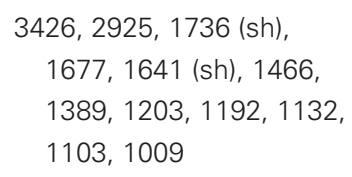 \\
\hline Color reaction positive: & $\begin{array}{l}\mathrm{I}_{2} \text {, vanillin-sulfuric acid } \\
\text { molybdophosphoric } \\
\text { acid-sulfuric acid }\end{array}$ & $\begin{array}{l}\mathrm{I}_{2} \text {, vanillin-sulfuric acid } \\
\text { molybdophosphoric } \\
\text { acid-sulfuric acid }\end{array}$ & $\begin{array}{l}I_{2} \text {, vanillin-sulfuric acid } \\
\text { molybdophosphoric } \\
\text { acid-sulfuric acid }\end{array}$ & $\begin{array}{l}\mathrm{I}_{2} \text {, vanillin-sulfuric acid } \\
\text { molybdophosphoric } \\
\text { acid-sulfuric acid }\end{array}$ \\
\hline \multirow[t]{3}{*}{ Silica gel TLC } & $\operatorname{Rf} 0.19^{*} \operatorname{Rf} 0.04^{* *}$ & $\operatorname{Rf} 0.19 * \operatorname{Rf} 0.04^{* *}$ & $\operatorname{Rf} 0.19 * \operatorname{Rf} 0.04^{* *}$ & $\operatorname{Rf} 0.19 * \operatorname{Rf} 0.04 * *$ \\
\hline & $\operatorname{Rf} 0.44^{* * *}$ & $\operatorname{Rf} 0.44^{* * *}$ & $\operatorname{Rf} 0.44^{* * *}$ & $\operatorname{Rf} 0.44^{* * *}$ \\
\hline & Caprazamycin E (5) & Caprazamycin F (6) & Caprazamycin G (7) & \\
\hline Appearance & colorless powder & colorless powder & colorless powder & \\
\hline Molecular formula & $\begin{array}{l}\mathrm{C}_{51} \mathrm{H}_{83} \mathrm{~N}_{5} \mathrm{O}_{22} \\
\text { [obsd. } 1118.5613 \\
\left.\quad(\mathrm{M}+\mathrm{H})^{+}, \text {error }+0.5\right]\end{array}$ & $\begin{array}{l}\mathrm{C}_{51} \mathrm{H}_{83} \mathrm{~N}_{5} \mathrm{O}_{22} \\
\text { [obsd. } 1118.5615 \\
\left.\quad(\mathrm{M}+\mathrm{H})^{+}, \text {error }+0.7\right]\end{array}$ & $\begin{array}{l}\mathrm{C}_{52} \mathrm{H}_{85} \mathrm{~N}_{5} \mathrm{O}_{22} \\
\text { [obsd. } 1132.5747 \\
\left.\quad(\mathrm{M}+\mathrm{H})^{+}, \text {error }-1.7\right]\end{array}$ & \\
\hline \multirow[t]{2}{*}{ FAB-MS $(m / z)$} & $1118(\mathrm{M}+\mathrm{H})^{+}$ & $1118(\mathrm{M}+\mathrm{H})^{+}$ & $1132(\mathrm{M}+\mathrm{H})^{+}$ & \\
\hline & $1116(\mathrm{M}-\mathrm{H})^{-}$ & $1116(\mathrm{M}-\mathrm{H})^{-}$ & $1130(\mathrm{M}-\mathrm{H})^{-}$ & \\
\hline$[\alpha]_{D}^{23}$ & -5.1 (c 0.83, DMSO) & -4.7 (c 0.90, DMSO) & $-4.2(c 1, \mathrm{MeOH})$ & \\
\hline UV $\lambda_{\max }^{\mathrm{MeOH}} \mathrm{nm}(\varepsilon)$ & $262(7,700)$ & $262(7,600)$ & $262(9,000)$ & \\
\hline $0.03 \mathrm{M} \mathrm{HCl}-\mathrm{MeOH}$ & $262(7,500)$ & $262(7,400)$ & $261(8,600)$ & \\
\hline $0.03 \mathrm{M} \mathrm{NaOH}-\mathrm{MeOH}$ & $261(5,900)$ & $261(5,800)$ & $260(7,000)$ & \\
\hline $\mathrm{IR} v_{\max }(\mathrm{KBr}) \mathrm{cm}^{-1}$ & $\begin{array}{l}3421,2925,2854,1739 \\
\text { 1697, } 1675 \text { (sh), 1629, } \\
\text { 1465, 1386, 1268, } \\
\text { 1124, } 1103\end{array}$ & $\begin{array}{l}3450,2929,2856,1737 \\
\text { 1704, 1631, 1465, } \\
\text { 1386, 1268, 1122, } \\
11031\end{array}$ & $\begin{array}{c}3423,2929,2854,1738 \\
1680,1637 \text { (sh), } 1466 \\
1392,1271,1203 \\
1184,1132,1009\end{array}$ & \\
\hline Color reaction positive: & $\begin{array}{l}\mathrm{I}_{2} \text {, vanillin-sulfuric acid } \\
\text { molybdophosphoric } \\
\text { acid-sulfuric acid }\end{array}$ & $\begin{array}{l}\mathrm{I}_{2} \text {, vanillin-sulfuric acid } \\
\text { molybdophosphoric } \\
\text { acid-sulfuric acid }\end{array}$ & $\begin{array}{l}\mathrm{I}_{2} \text {, vanillin-sulfuric acid } \\
\text { molybdophosphoric } \\
\text { acid-sulfuric acid }\end{array}$ & \\
\hline Silica gel TLC & $\begin{array}{l}\operatorname{Rf} 0.19 * \operatorname{Rf} 0.04 * * \\
\quad \operatorname{Rf} 0.44^{* * *}\end{array}$ & $\begin{array}{l}\operatorname{Rf} 0.19 * \operatorname{Rf} 0.04^{* *} \\
\quad \operatorname{Rf} 0.44^{* * *}\end{array}$ & $\begin{array}{l}\operatorname{Rf} 0.19^{*} \operatorname{Rf} 0.04^{* *} \\
\quad \operatorname{Rf} 0.44^{* * *}\end{array}$ & \\
\hline
\end{tabular}

* Kieselgel $60 \mathrm{~F}_{254}$, art 5715, Merck $\left(\mathrm{CHCl}_{3}: \mathrm{MeOH}: \mathrm{H}_{2} \mathrm{O}:\right.$ formic acid=10:5:1:0.1), ** $\left(\mathrm{CHCl}_{3}: \mathrm{MeOH}: \mathrm{H}_{2} \mathrm{O}: \mathrm{conc} \mathrm{NH}_{4} \mathrm{OH}=10: 5: 1: 0.1\right)$, $* * *\left(\mathrm{BuOH}: \mathrm{MeOH}: \mathrm{H}_{2} \mathrm{O}=4: 1: 2\right)$

caprazene, and the diacid 12 in high yield, respectively. The core component $\mathbf{1 1}$ was also obtained quantitatively by direct hydrolysis of $\mathbf{2}$ under the same conditions. The two carboxyl groups of $\mathbf{1 2}$ were treated with 4-bromoaniline in the presence of bis(2-oxo-3-oxazolidinyl)phosphinic chloride (BOP-Cl) to give the bromoanilide $\mathbf{1 3}$ in $70 \%$ yield, which was crystallized from its acetone solution. The ORTEP drawing of $\mathbf{1 3}$ is shown in Fig. 4. On the basis of 
Table $2{ }^{13} \mathrm{C}$ NMR data of CPZs (125 MHz)

\begin{tabular}{|c|c|c|c|c|c|c|}
\hline $1^{*}$ & $2^{* *}$ & $\mathbf{3}^{*}$ & $\mathbf{4}^{*}$ & $\mathbf{5}^{* *}$ & $\begin{array}{l}\mathbf{6}^{*} \\
\delta\end{array}$ & $\mathbf{7}^{*}$ \\
\hline$\delta$ & $\delta$ & $\delta$ & $\delta$ & & & \\
\hline $13.9 \mathrm{q}$ & $17.8 \mathrm{q}$ & $13.9 \mathrm{q}$ & $18.2 \mathrm{q}$ & $14.1 \mathrm{q}$ & $17.7 \mathrm{q}$ & $11.8 \mathrm{q}$ \\
\hline $17.7 \mathrm{q}$ & $19.2 \mathrm{q}$ & $17.7 \mathrm{q}$ & $20.2 \mathrm{q}$ & $17.9 \mathrm{q}$ & $19.0 \mathrm{q}$ & $18.2 \mathrm{q}$ \\
\hline $19.0 \mathrm{q}$ & $22.6 \mathrm{q}$ & $19.0 \mathrm{q}$ & $23.0 \mathrm{q}$ & $19.3 q$ & $22.5 \mathrm{q}$ & $19.6 \mathrm{q}$ \\
\hline $22.1 \mathrm{t}$ & $22.6 \mathrm{q}$ & $22.1 \mathrm{t}$ & $23.0 \mathrm{q}$ & $22.3 t$ & $24.5 t$ & $20.1 \mathrm{q}$ \\
\hline $24.5 t$ & $24.6 t$ & $24.5 \mathrm{t}$ & $26.3 t$ & $24.7 t$ & $26.7 t$ & $26.3 t$ \\
\hline $27.1 \mathrm{~d}$ & $26.9 t$ & $27.1 \mathrm{~d}$ & $28.5 t$ & $28.8 \mathrm{t}$ & $27.4 \mathrm{t}$ & $28.2 \mathrm{t}$ \\
\hline $28.6 t$ & $27.2 \mathrm{~d}$ & $28.7 t$ & $30.4 \mathrm{~d}$ & $27.3 d$ & $27.1 \mathrm{~d}$ & $28.8 d$ \\
\hline $28.7 t$ & $27.5 \mathrm{~d}$ & $28.6 t$ & $28.7 \mathrm{~d}$ & $29.0 \mathrm{t}$ & $28.7 t$ & $30.4 d$ \\
\hline $28.7 t$ & $28.7 t$ & $28.7 t$ & $29.1 t$ & $28.8 t$ & $28.6 t$ & $30.6 \mathrm{t}$ \\
\hline $28.8 t$ & $28.9 t$ & $28.9 t$ & $30.6 \mathrm{t}$ & $29.0 t$ & $28.9 t$ & $30.6 \mathrm{t}$ \\
\hline $28.9 t$ & $29.0 t$ & $29.0 t$ & $30.6 \mathrm{t}$ & $29.1 \mathrm{t}$ & $28.9 t$ & $30.7 \mathrm{t}$ \\
\hline $28.9 t$ & $29.1 t$ & $29.0 t$ & $30.8 t$ & $29.1 \mathrm{t}$ & $29.0 t$ & $31.0 \mathrm{t}$ \\
\hline $29.0 t$ & $29.1 \mathrm{t}$ & $29.0 t$ & $31.0 \mathrm{t}$ & $31.5 t$ & $29.2 t$ & $35.2 \mathrm{t}$ \\
\hline $29.0 t$ & $29.4 t$ & $31.3 t$ & $35.3 t$ & $33.4 \mathrm{t}$ & $33.2 \mathrm{t}$ & $35.7 t$ \\
\hline $31.2 \mathrm{t}$ & $33.3 t$ & $33.2 \mathrm{t}$ & $39.0 \mathrm{q}$ & $36.1 \mathrm{q}$ & $36.2 \mathrm{q}$ & $37.6 \mathrm{q}$ \\
\hline $33.2 \mathrm{t}$ & $36.1 \mathrm{q}$ & $35.8 q$ & $39.3 \mathrm{q}$ & $37.6 q$ & $37.7 \mathrm{q}$ & $37.8 \mathrm{q}$ \\
\hline $35.8 \mathrm{q}$ & $37.5 \mathrm{q}$ & $37.3 q$ & $40.2 \mathrm{t}$ & $38.8 \mathrm{t}$ & $38.4 \mathrm{t}$ & $38.6 \mathrm{t}$ \\
\hline $37.3 q$ & $38.6 t$ & $38.6 t$ & $40.3 t$ & $40.2 t$ & $38.8 t$ & $40.3 \mathrm{t}$ \\
\hline $38.6 t$ & $38.7 t$ & $40.0 t$ & $41.4 \mathrm{t}$ & $40.2 t$ & $40.0 t$ & $41.4 \mathrm{t}$ \\
\hline $40.0 \mathrm{t}$ & $39.5 t$ & $40.0 \mathrm{t}$ & $41.6 \mathrm{t}$ & $40.3 t$ & $40.1 \mathrm{t}$ & $41.7 \mathrm{t}$ \\
\hline $40.0 t$ & $40.2 t$ & $40.1 \mathrm{t}$ & $42.6 \mathrm{t}$ & $56.7 t$ & $40.1 \mathrm{q}$ & $42.1 \mathrm{t}$ \\
\hline $40.2 \mathrm{t}$ & $40.4 t$ & $56.5 t$ & $58.0 t$ & $56.9 q$ & $56.5 d$ & $58.0 \mathrm{t}$ \\
\hline $56.5 \mathrm{t}$ & $56.7 \mathrm{t}$ & $56.7 \mathrm{q}$ & $59.0 \mathrm{q}$ & $58.5 q$ & $56.7 d$ & $58.0 \mathrm{q}$ \\
\hline $56.6 \mathrm{q}$ & $56.8 \mathrm{q}$ & $58.3 \mathrm{q}$ & $59.3 \mathrm{q}$ & $60.3 \mathrm{q}$ & $58.3 d$ & $59.3 \mathrm{q}$ \\
\hline $58.3 \mathrm{q}$ & $58.5 \mathrm{q}$ & $60.1 \mathrm{q}$ & $61.2 \mathrm{q}$ & $63.1 \mathrm{~d}$ & $60.1 \mathrm{~d}$ & $61.2 \mathrm{q}$ \\
\hline $60.1 \mathrm{q}$ & $60.2 \mathrm{q}$ & $62.9 \mathrm{~d}$ & $64.3 \mathrm{~d}$ & $63.2 \mathrm{~d}$ & $62.6 \mathrm{~d}$ & $64.8 d$ \\
\hline $62.9 \mathrm{~d}$ & $62.9 \mathrm{~d}$ & $63.0 \mathrm{~d}$ & $64.7 \mathrm{~d}$ & $69.0 \mathrm{~d}$ & $68.9 \mathrm{~d}$ & $65.0 d$ \\
\hline $63.0 \mathrm{~d}$ & $63.0 \mathrm{~d}$ & $68.8 d$ & $69.9 \mathrm{~d}$ & $69.7 \mathrm{~d}$ & $69.5 \mathrm{~d}$ & $70.7 d$ \\
\hline $68.8 \mathrm{~d}$ & $68.9 \mathrm{~d}$ & $69.5 \mathrm{~d}$ & $70.6 \mathrm{~d}$ & $70.0 \mathrm{~d}$ & $69.9 \mathrm{~d}$ & $71.4 d$ \\
\hline $69.5 \mathrm{~d}$ & $69.6 \mathrm{~d}$ & $69.8 d$ & $71.3 \mathrm{~d}$ & $70.4 \mathrm{~d}$ & $72.2 \mathrm{~d}$ & $71.9 d$ \\
\hline $69.8 \mathrm{~d}$ & $69.9 \mathrm{~d}$ & $74.1 \mathrm{~d}$ & $75.2 \mathrm{~d}$ & $70.3 d$ & $70.2 \mathrm{~d}$ & $75.7 d$ \\
\hline $70.3 d$ & $70.0 \mathrm{~d}$ & $70.2 d$ & $71.9 \mathrm{~d}$ & $74.5 d$ & $74.4 \mathrm{~d}$ & $72.2 \mathrm{~d}$ \\
\hline $70.3 d$ & $70.3 d$ & $70.2 \mathrm{~d}$ & $72.2 \mathrm{~d}$ & $74.2 \mathrm{~d}$ & $74.3 \mathrm{~d}$ & $72.4 d$ \\
\hline $74.2 \mathrm{~d}$ & $74.2 \mathrm{~d}$ & $74.5 \mathrm{~d}$ & $75.7 d$ & $75.3 d$ & $76.5 d$ & $76.4 d$ \\
\hline $74.5 \mathrm{~d}$ & $74.5 \mathrm{~d}$ & $78.2 \mathrm{~d}$ & $80.8 d$ & $75.2 d$ & $75.1 \mathrm{~d}$ & $80.2 d$ \\
\hline $75.1 \mathrm{~d}$ & $75.1 \mathrm{~d}$ & $75.1 \mathrm{~d}$ & $75.9 \mathrm{~d}$ & $80.2 \mathrm{~d}$ & $80.1 \mathrm{~d}$ & $77.2 \mathrm{~d}$ \\
\hline $75.1 \mathrm{~d}$ & $75.3 d$ & $75.1 \mathrm{~d}$ & $77.4 \mathrm{~d}$ & $78.3 d$ & $78.3 d$ & $77.4 \mathrm{~d}$ \\
\hline $78.2 \mathrm{~d}$ & $78.3 d$ & $80.1 d$ & $82.1 \mathrm{~d}$ & $81.1 \mathrm{~d}$ & $80.9 \mathrm{~d}$ & $82.1 d$ \\
\hline $80.1 \mathrm{~d}$ & $80.2 \mathrm{~d}$ & $80.9 d$ & $82.8 d$ & $82.3 d$ & $83.0 \mathrm{~d}$ & $82.8 d$ \\
\hline $80.8 d$ & $81.0 \mathrm{~d}$ & $82.0 \mathrm{~d}$ & $83.8 \mathrm{~d}$ & $89.4 d$ & $88.9 \mathrm{~d}$ & $83.9 \mathrm{~d}$ \\
\hline $82.1 \mathrm{~d}$ & $82.2 \mathrm{~d}$ & $89.2 \mathrm{~d}$ & $92.3 \mathrm{~d}$ & $90.7 d$ & $90.5 d$ & $92.3 \mathrm{~d}$ \\
\hline $89.2 d$ & $89.3 d$ & $90.5 d$ & $92.9 \mathrm{~d}$ & $101.3 d$ & $101.1 \mathrm{~s}$ & $92.5 d$ \\
\hline $90.4 \mathrm{~d}$ & $90.6 \mathrm{~d}$ & $101.1 d$ & $102.8 d$ & $110.2 \mathrm{~d}$ & $110.0 \mathrm{~s}$ & $102.2 d$ \\
\hline $101.1 d$ & $101.3 \mathrm{~d}$ & $110.1 d$ & $110.8 d$ & $140.0 \mathrm{~d}$ & $140.2 \mathrm{~s}$ & $111.9 d$ \\
\hline $110.1 d$ & $110.1 \mathrm{~d}$ & $139.7 d$ & $142.8 d$ & $150.4 \mathrm{~s}$ & $150.3 \mathrm{~s}$ & $142.4 d$ \\
\hline $139.7 d$ & $139.9 \mathrm{~d}$ & $150.2 \mathrm{~s}$ & $152.1 \mathrm{~s}$ & $163.6 \mathrm{~s}$ & $163.3 \mathrm{~s}$ & $152.0 \mathrm{~s}$ \\
\hline $150.2 \mathrm{~s}$ & $150.3 \mathrm{~s}$ & $163.3 \mathrm{~s}$ & $166.1 \mathrm{~s}$ & $169.3 \mathrm{~s}$ & $169.2 \mathrm{~s}$ & $166.2 \mathrm{~s}$ \\
\hline $163.2 \mathrm{~s}$ & $163.5 \mathrm{~s}$ & $169.0 \mathrm{~s}$ & $168.0 \mathrm{~s}$ & $169.8 \mathrm{~s}$ & $169.2 \mathrm{~s}$ & $171.0 \mathrm{~s}$ \\
\hline $169.0 \mathrm{~s}$ & $169.1 \mathrm{~s}$ & $169.7 \mathrm{~s}$ & $170.1 \mathrm{~s}$ & $170.1 \mathrm{~s}$ & $170.2 \mathrm{~s}$ & $171.0 \mathrm{~s}$ \\
\hline $169.7 \mathrm{~s}$ & $169.6 \mathrm{~s}$ & $170.0 \mathrm{~s}$ & $170.4 \mathrm{~s}$ & $170.6 \mathrm{~s}$ & $170.4 \mathrm{~s}$ & $172.3 \mathrm{~s}$ \\
\hline $170.1 \mathrm{~s}$ & $170.0 \mathrm{~s}$ & $170.3 \mathrm{~s}$ & $172.3 \mathrm{~s}$ & $171.5 \mathrm{~s}$ & $171.1 \mathrm{~s}$ & $172.3 \mathrm{~s}$ \\
\hline $170.2 \mathrm{~s}$ & $170.5 \mathrm{~s}$ & $171.2 \mathrm{~s}$ & $173.7 \mathrm{~s}$ & $172.3 \mathrm{~s}$ & & $173.6 \mathrm{~s}$ \\
\hline $171.2 \mathrm{~s}$ & $171.4 \mathrm{~s}$ & & & & & \\
\hline
\end{tabular}

${ }^{*}$ DMSO $-d_{6,}{ }^{*}$ DMSO $-d_{6}-\mathrm{D}_{2} \mathrm{O}(10: 1)$

$\mathrm{X}$-ray structure analysis of $\mathbf{1 3}$, the configurations at $\mathrm{C}-3 \mathrm{a}$ and C-3b of caprazamycin B (2) were determined to be $S$ and $S$, respectively.
Alkaline degradation studies of $\mathbf{2}$ also gave another core component. Treatment of $\mathbf{2}$ with aqueous ammonia in $\mathrm{N}, \mathrm{N}$ dimethylformamide for $\mathbf{4}$ days at room temperature gave the deacylated compound $\mathbf{1 4}$ quantitatively, which was designated as caprazol (Scheme 2). Crystallization of $\mathbf{1 4}$ from aqueous methanol afforded prisms suitable for X-ray structure analysis. The ORTEP drawing of caprazol (14) is shown in Fig. 5. To elucidate the absolute structure of the ribose moiety of $\mathbf{2}$, the reference methyl 5-amino-5-deoxy$\alpha$ - and $\beta$-D-ribofuranosides (18a and $\mathbf{1 8 b}$ ) were prepared from 5-azido-5-deoxy-1,2- $O$-isopropylidene- $\alpha$-D-ribose (15) [10] in two steps (Scheme 3). Methanolysis of 15 in the presence of cation-exchange resin gave an anomeric mixture (1:2.9) of methyl glycosides (16a and 16b) in 63\% yield, along with methyl 5-azido-5-deoxy-2,3-Oisopropylidene- $\beta$-D-ribofuranoside (17) $[10,11]$. Treatment of a mixture of $16 \mathrm{a}$ and $16 \mathrm{~b}$ with triphenylphospine in aqueous tetrahydrofuran gave an anomeric mixture of the free amino sugars (18a and 18b), quantitatively. Separation of these anomers was successfully performed by silica gel column chromatography.

On the other hand, caprazene (11) was solvolyzed in boiling methanol in the presence of cation-exchange resin for 14 hours to give a mixture $(1: 2.2)$ of $\mathbf{1 8 a}$ and $\mathbf{1 8 b}$ in $51 \%$ yield. The $\beta$-glycoside $\mathbf{1 8 b}$ isolated, was in all respects identical with the reference sample prepared from 15. The agreement on the specific rotation value between the both compounds proved that the 5-amino-5-deoxyribose moiety in caprazamycin B (2) is the D-sugar.

Finally, based on the results described above, the stereochemistry of 2 was established to be C-5' $(S), \mathrm{C}-2^{\prime \prime \prime}$ $(S), \mathrm{C}-3^{\prime \prime \prime}(S), \mathrm{C}-6^{\prime \prime \prime}(S), \mathrm{C}-3 \mathrm{a}(S)$ and $\mathrm{C}-3 \mathrm{~b}(S)$ as shown in Fig. 1.

The CPZs are structurally related to lipo-uridyl antibiotics such as liposidomycins, muraymycins [12] and capuramycins [13 15], which have been shown to be specific inhibitors of a bacterial translocase. Liposidomycins are especially, close analogs of $\mathrm{CPZs}$, differing in the absence of the tri- $O$-methyl-L-rhamnose moiety and the dissimilarity for configuration of the acylated side chain at the C-3a position [5]. These nucleoside antibiotics comprise a novel type of drugs having the inhibitory activity against bacterial cell wall biosynthesis.

In the degradation studies of caprazamycins, we obtained efficiently the two core components, caprazene (11) and caprazol (14). These compounds were considered to be attractive precursors for the synthesis of caprazamycin analogs. Semi-synthetic antibiotics derived from $\mathbf{1 1}$ or $\mathbf{1 4}$ and their biological activities will be reported later. 
Table $3{ }^{13} \mathrm{C}$ and ${ }^{1} \mathrm{H}$ NMR data of caprazamycin B (2) in DMSO- $d_{6}$ - pyridine- $d_{5}-\mathrm{D}_{2} \mathrm{O}(5: 5: 1)$

\begin{tabular}{|c|c|c|c|c|c|c|c|}
\hline No. & $\delta_{\mathrm{C}}{ }^{*}$ & $\delta_{\mathrm{H}}^{* *}$ & $J(\mathrm{~Hz})$ & No. & $\delta_{\mathrm{C}}{ }^{*}$ & $\delta_{\mathrm{H}}^{* *}$ & $J(\mathrm{~Hz})$ \\
\hline 2 & $150.7 \mathrm{~s}$ & & & $6 a^{* * *}$ & $29.5 t$ & $2.61 \mathrm{~m}$ & \\
\hline 4 & $163.9 \mathrm{~s}$ & & & $7 a^{* * *}$ & $29.5 t$ & $2.61 \mathrm{~m}$ & \\
\hline 5 & $101.7 d$ & $5.97 d$ & 8 & $8 a^{* * *}$ & $29.4 t$ & $2.61 \mathrm{~m}$ & \\
\hline 6 & $140.0 \mathrm{~d}$ & $8.07 \mathrm{~d}$ & 8 & $9 a^{* * *}$ & $29.4 t$ & $2.61 \mathrm{~m}$ & \\
\hline $1^{\prime}$ & $90.2 d$ & $5.84 \mathrm{~d}$ & 1 & $10 a^{* * *}$ & $29.2 \mathrm{t}$ & $2.61 \mathrm{~m}$ & \\
\hline $2^{\prime}$ & $75.4 \mathrm{~d}$ & $4.32 \mathrm{dd}$ & 1,4 & $11 a$ & $29.8 t$ & $2.61 \mathrm{~m}$ & \\
\hline $3^{\prime}$ & $69.7 \mathrm{~d}$ & $4.37 \mathrm{dd}$ & 4,8 & $12 a$ & $27.3 t$ & $1.26 \mathrm{~m}$ & \\
\hline $4^{\prime}$ & $83.0 \mathrm{~d}$ & $4.77 \mathrm{dd}$ & 2,4 & $13 a$ & $38.6 t$ & $1.13 \mathrm{~m}$ & \\
\hline $5^{\prime}$ & $76.2 \mathrm{~d}$ & $4.71 \mathrm{dd}$ & 2,9 & $14 a$ & $27.8 d$ & $1.48 \mathrm{~m}$ & \\
\hline $1^{\prime \prime}$ & $111.1 \mathrm{~d}$ & $5.62 \mathrm{~s}$ & $\sim 1$ & 14a-Me & $22.6 \mathrm{q}$ & $1.86 \mathrm{~d}$ & 6 \\
\hline $2^{\prime \prime}$ & $75.1 \mathrm{~d}$ & $4.43 d$ & 4 & $15 a$ & $22.6 \mathrm{q}$ & $1.86 \mathrm{~d}$ & 6 \\
\hline $3^{\prime \prime}$ & $71.0 \mathrm{~d}$ & $4.65 \mathrm{dd}$ & 4,8 & $1 b$ & $171.7 \mathrm{~s}$ & & \\
\hline $4^{\prime \prime}$ & $79.1 \mathrm{~d}$ & $4.53 \mathrm{ddd}$ & $4,4,8$ & $2 b$ & $40.5 \mathrm{t}$ & $2.62 \mathrm{~m}$ & \\
\hline $5^{\prime \prime}$ & $40.5 t$ & $3.49 \mathrm{~m}$ & & & & $2.4 \mathrm{dd}$ & 7,14 \\
\hline $1^{\prime \prime \prime}$ & $170.8 \mathrm{~s}$ & & & $3 b$ & $27.6 \mathrm{~d}$ & $2.61 \mathrm{~m}$ & \\
\hline $2^{\prime \prime \prime}$ & $63.9 d$ & $4.94 \mathrm{~d}$ & 4.5 & $3 b-M e$ & $19.4 \mathrm{q}$ & $1.11 d$ & 5 \\
\hline $3^{\prime \prime \prime}$ & $71.4 \mathrm{~d}$ & $5.75 \mathrm{~m}$ & & $4 b$ & $40.6 \mathrm{t}$ & $2.64 \mathrm{~m}$ & \\
\hline \multirow[t]{2}{*}{$4^{\prime \prime \prime}$} & $57.1 \mathrm{t}$ & $3.83 \mathrm{~m}$ & & & & $2.47 \mathrm{dd}$ & 7,14 \\
\hline & & $3.68 \mathrm{~m}$ & & $5 b$ & $171.0 \mathrm{~s}$ & & \\
\hline $5^{\prime \prime \prime}-N M e$ & $36.4 \mathrm{q}$ & $2.72 \mathrm{~s}$ & & $1 \mathrm{c}$ & $91.2 \mathrm{~d}$ & $6.34 \mathrm{~d}$ & 2 \\
\hline $6^{\prime \prime \prime}$ & $63.8 \mathrm{~d}$ & $4.15 d$ & 9 & $2 c$ & $76.0 \mathrm{~d}$ & $3.83 \mathrm{~m}$ & \\
\hline $7^{\prime \prime \prime}$ & $170.8 \mathrm{~s}$ & & & 2c-OMe & $57.1 \mathrm{q}$ & $3.50 \mathrm{~s}$ & \\
\hline $8^{\prime \prime \prime}-N M e$ & $37.9 \mathrm{q}$ & $3.35 \mathrm{~s}$ & & $3 c$ & $80.9 d$ & $3.67 \mathrm{dd}$ & 3,9 \\
\hline $1 a$ & $169.7 \mathrm{~s}$ & & & 3c-OMe & $58.8 \mathrm{q}$ & $3.53 \mathrm{~s}$ & \\
\hline $2 a$ & $39.2 \mathrm{t}$ & $2.88 \mathrm{~m}$ & & $4 c$ & $81.6 \mathrm{~d}$ & $3.33 \mathrm{dd}$ & 9,9 \\
\hline $3 a$ & $70.5 d$ & $5.48 \mathrm{~m}$ & & 4c-OMe & $60.5 q$ & $3.56 \mathrm{~s}$ & \\
\hline $4 a$ & $33.9 t$ & $1.75 \mathrm{~m}$ & & $5 c$ & $70.2 \mathrm{~d}$ & $3.83 \mathrm{~m}$ & \\
\hline $5 a$ & $25.1 \mathrm{t}$ & $1.40 \mathrm{~m}$ & & $6 c$ & $18.0 \mathrm{q}$ & $1.32 \mathrm{~d}$ & 6 \\
\hline
\end{tabular}

* $125 \mathrm{MHz}$, chemical shifts in ppm, multiplicity. ${ }^{*} 500 \mathrm{MHz}$, chemical shifts in ppm, multiplicity.

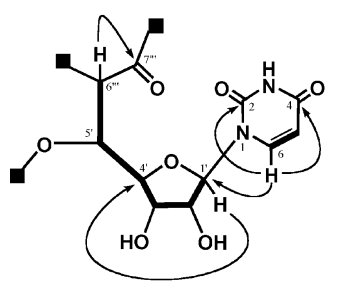

a

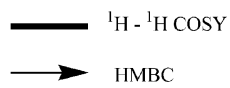

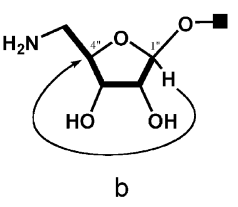

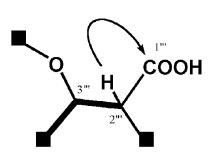

C

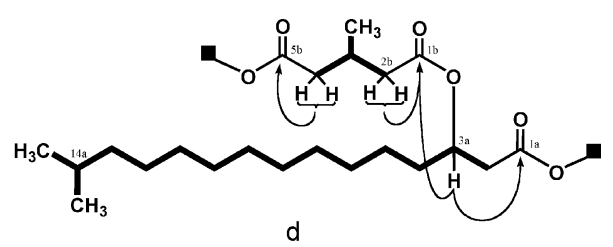

d
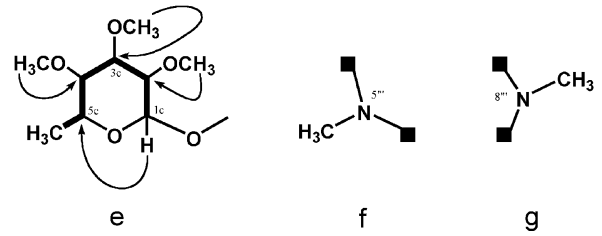

Fig. 2 Partial structures of caprazamycin B (2). 


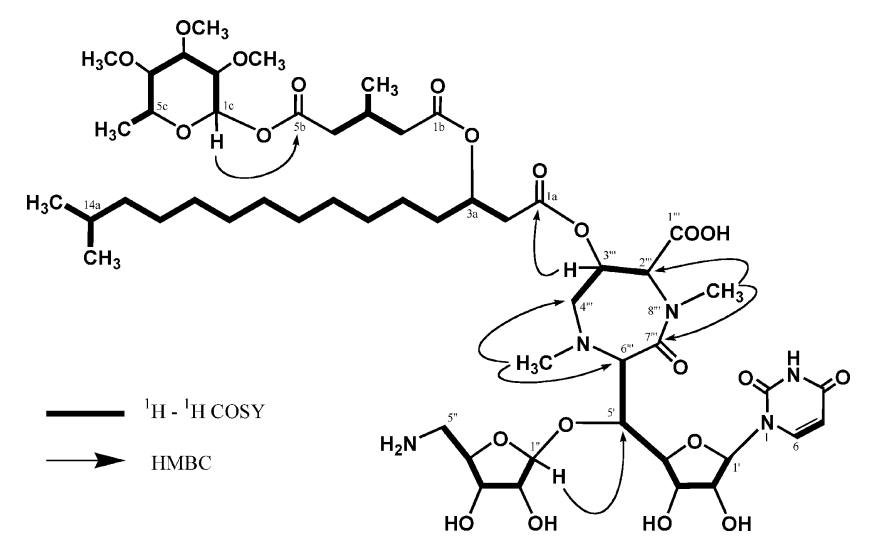

Fig. $3 \mathrm{HMBC}$ correlations of caprazamycin $B$ (2) in DMSO- $d_{6}$ - pyridine $-d_{5}-\mathrm{D}_{2} \mathrm{O}(5: 5: 1)$.
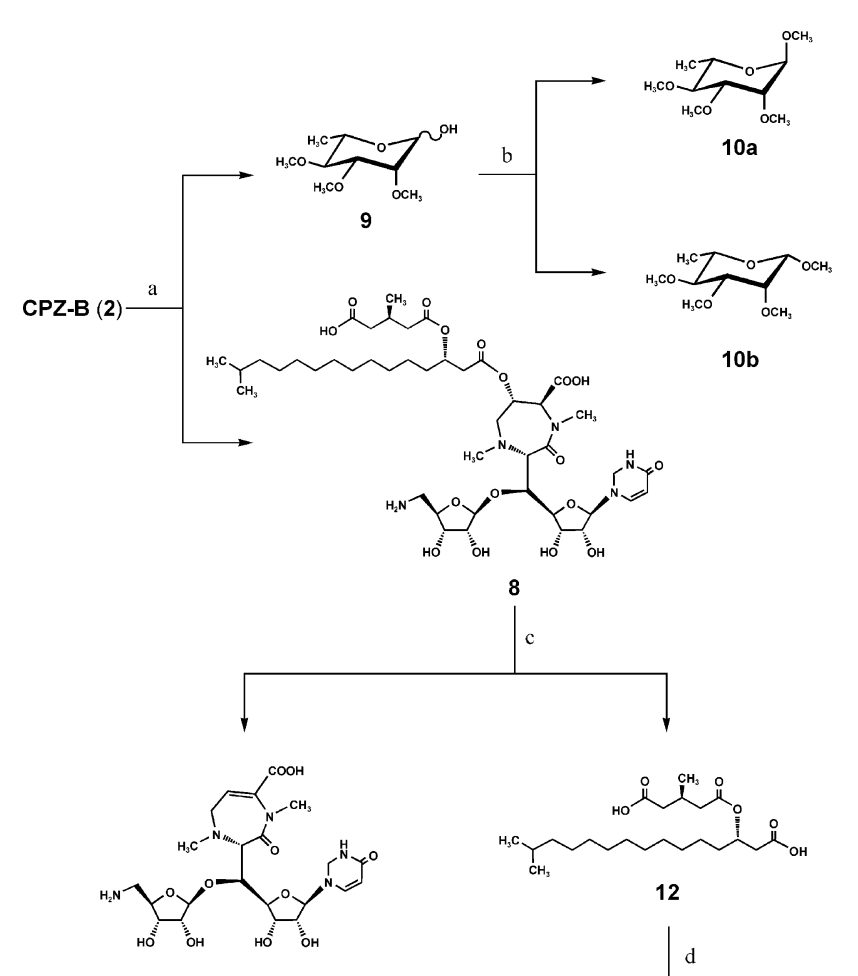

caprazene (11)
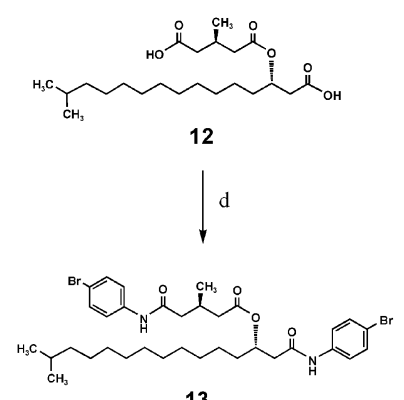

Conditions: (a) $80 \% \mathrm{CF}_{3} \mathrm{COOH}, \mathrm{MeOH}$, rt, 1 hour, 8: 99\%, 9: 97\%; (b) $\mathrm{H}_{2} \mathrm{SO}_{4}, \mathrm{MeOH}, 50^{\circ} \mathrm{C}$, 4 hours, 10a $+10 b$ (5:1): $87 \%$; (c) $80 \%$ aq $\mathrm{AcOH}, 70{ }^{\circ} \mathrm{C}, 2$ hours, 11: $99 \%$, 12: $92 \%$; (d) 4-bromoaniline, BOP-Cl, $\mathrm{Et}_{3} \mathrm{~N}, \mathrm{THF}, \mathrm{rt}, 2$ hours, $70 \%$.

Scheme 1 Degradation study of caprazamycin B (2).

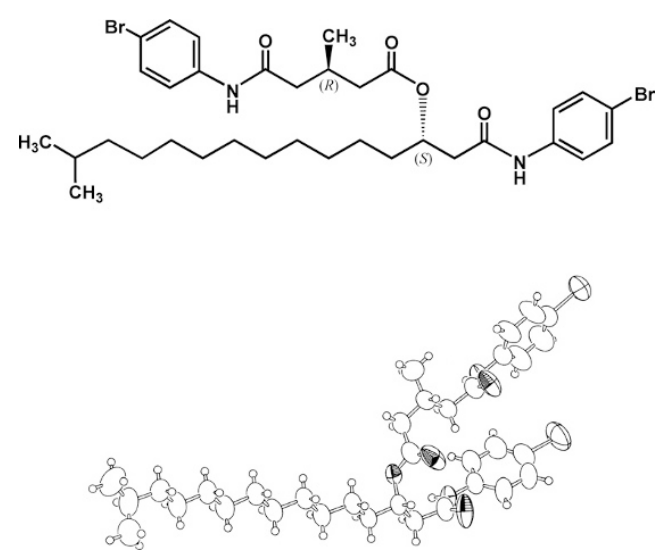

Fig. 4 Structure of $\mathbf{1 3}$ and its ORTEP.

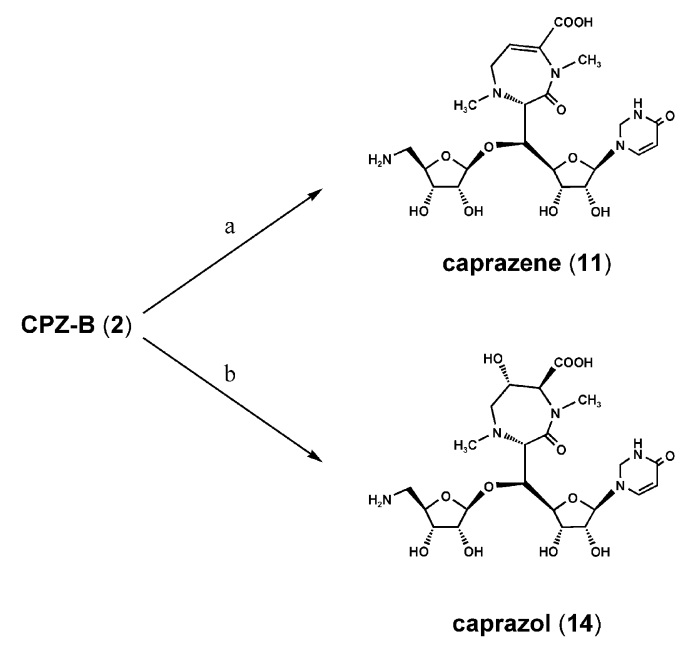

Conditions: (a) $80 \%$ aq $\mathrm{AcOH}, 70^{\circ} \mathrm{C}, 2$ hours, $99 \%$; (b) $28 \%$ aq $\mathrm{NH}_{3}$, $\mathrm{DMF}$, rt, 4 days, $99 \%$.

Scheme 2 Synthesis of the core components of caprazamycins.
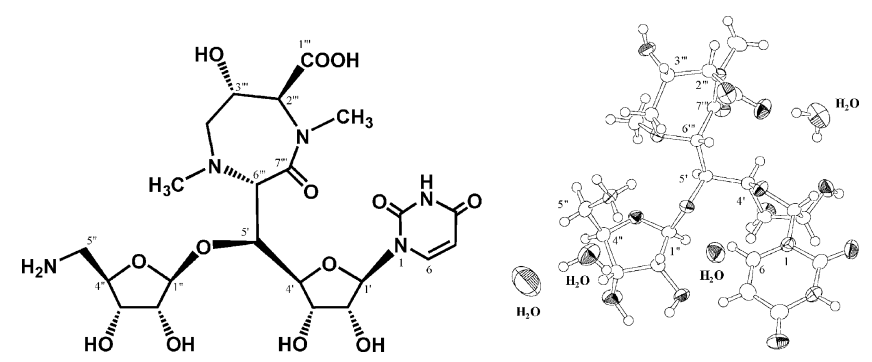

Fig. 5 Structure of caprazol (14) and its ORTEP. 


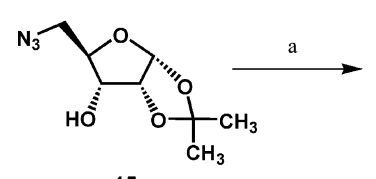

15

11

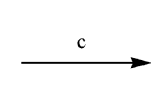

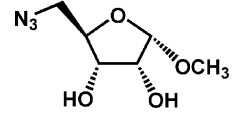

$16 a$

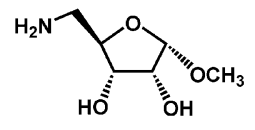

$18 \mathrm{a}$

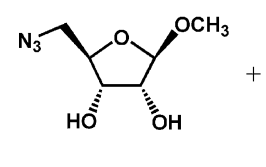

$16 b$

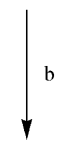

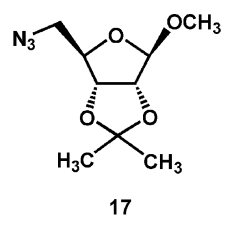

17

Conditions: (a) Amberlite CG $120\left(\mathrm{H}^{+}\right.$), MeOH, reflux, 30 minutes, 16a+16b (1:2.9): 63\%, 17:22\%; (b) $\mathrm{Ph}_{3} \mathrm{P}, \mathrm{THF}-\mathrm{H}_{2} \mathrm{O}$, rt, overnight, 99\%; (c)

Amberlite $\mathrm{CG} 120\left(\mathrm{H}^{+}\right), \mathrm{MeOH}$, reflux, 14 hours, 18a+18b (1:2.2): 51\%.

Scheme 3 Synthesis of methyl 5-amino-5-deoxy- $\alpha$ - and $\beta$-D-ribofuranoside (18a and $\mathbf{1 8 b}$ ).

\section{Experimental}

\section{General Methods}

Melting points were determined on a Kofler block and are uncorrected. Optical rotations were determined with a Perkin-Elmer 241 polarimeter. UV spectra were measured with a Hitachi 557 spectrophotometer. IR spectra were recorded on a Horiba FT-210 Fourier transform infrared spectrometer. ${ }^{1} \mathrm{H}$ and ${ }^{13} \mathrm{C}$ NMR spectra were measured with a JEOL JNM-A500 and/or Bruker AVANCE 500 spectrometer, using TMS as an internal reference. Mass spectra were recorded using a JEOL JMS-SX102 (HRFAB) and/or JEOL JMS-T100LC (HR-ESI) spectrometer. $\mathrm{X}$-Ray crystallographic measurements were made on Rigaku AFC7R diffractometer with graphite monochromated $\mathrm{Cu}-\mathrm{K} \alpha$ radiation and a rotation anode generator. All calculations of the measurements were performed using the teXsan crystallographic software package of Molecular Structure Corporation. TLC was performed on Kieselgel $60 \mathrm{~F}_{254}$ (Merck), and column chromatography was carried out on Kieselgel 60 (Merck).

\section{Caprazamycin B1 (8) and 2,3,4-tri- $O$-methyl-L- rhamnose (9)}

A solution of $2(301 \mathrm{mg}, 0.263 \mathrm{mmol})$ in $4.5 \mathrm{ml}$ of trifluoroacetic acid-MeOH $(4: 1)$ was kept for 1 hour at room temparature. Concentration gave a syrup, which was added to diethyl ether. The precipitate obtained was filtered and thoroughly washed with diethyl ether to give a colorless solid of 8 (278.4 mg, $99 \%$ as the mono $\mathrm{CF}_{3} \mathrm{COOH}$ salt). The filtrate and washings were combined and concentrated to give $\mathbf{9}(52.5 \mathrm{mg}, 97 \%)$ as a syrup.
8: $[\alpha]_{\mathrm{D}}^{20}+12^{\circ}(c$ 0.5, DMSO); HR-MS (ESI, positive): $m / z$ 958.4918 $(\mathrm{M}+\mathrm{H})^{+}$(calcd for $\mathrm{C}_{44} \mathrm{H}_{72} \mathrm{~N}_{5} \mathrm{O}_{18}, 958.4872$ ); ${ }^{1} \mathrm{H}$ NMR $\left(500 \mathrm{MHz}\right.$, DMSO- $\left.d_{6}\right) \delta 0.84(6 \mathrm{H}, \mathrm{d}, J=7 \mathrm{~Hz}$, $\left.14 \mathrm{a}-\mathrm{Me}_{2}\right), 0.91$ (3H, d, $\left.J=6 \mathrm{~Hz}, 3 \mathrm{~b}-\mathrm{Me}\right), 1.44(1 \mathrm{H}, \mathrm{m}, 14 \mathrm{a}-$ $\mathrm{H}), 1.57\left(2 \mathrm{H}\right.$, br s, $\left.4 \mathrm{a}-\mathrm{H}_{2}\right), 2.26\left(3 \mathrm{H}, \mathrm{s}, 5^{\prime \prime \prime}-\mathrm{NMe}\right), 2.94(3 \mathrm{H}$, s, $\left.8^{\prime \prime \prime}-N \mathrm{Me}\right), 5.03\left(1 \mathrm{H}, \mathrm{s}, 1^{\prime \prime}-\mathrm{H}\right), 5.13(1 \mathrm{H}, \mathrm{m}, 3 \mathrm{a}-\mathrm{H}), 5.37$ $\left(1 \mathrm{H}\right.$, br s, $\left.3^{\prime \prime \prime}-\mathrm{H}\right), 5.56\left(1 \mathrm{H}, \mathrm{d}, J=\sim 1.5 \mathrm{~Hz}, 1^{\prime}-\mathrm{H}\right), 5.64(1 \mathrm{H}$, d, $J=8 \mathrm{~Hz}, 5-\mathrm{H}), 7.80(1 \mathrm{H}, \mathrm{d}, J=8 \mathrm{~Hz}, 6-\mathrm{H}), 11.30(1 \mathrm{H}, \mathrm{br}$, 3-NH).

9: ${ }^{1} \mathrm{H}$ NMR $\left(500 \mathrm{MHz}, \mathrm{CDCl}_{3}\right) \alpha$-isomer: $\delta 1.30(3 \mathrm{H}, \mathrm{d}$, $J=6 \mathrm{~Hz}, 5-\mathrm{Me}), 3.15(1 \mathrm{H}, \mathrm{t}, J=9.5$ and $9.5 \mathrm{~Hz}, 4-\mathrm{H}), 3.51$ $(6 \mathrm{H}, \mathrm{s}, \mathrm{OMe} \times 2), \sim 3.55(1 \mathrm{H}, 3-\mathrm{H}), 3.56(3 \mathrm{H}, \mathrm{s}, \mathrm{OMe})$, $3.62(1 \mathrm{H}, \mathrm{dd}, J=2$ and $3 \mathrm{~Hz}, 2-\mathrm{H}), 3.81(1 \mathrm{H}, \mathrm{dq}, J=6,6,6$ and $9.5 \mathrm{~Hz}, 5-\mathrm{H}), 5.27(1 \mathrm{H}, \mathrm{d}, J=1.8 \mathrm{~Hz}, 1-\mathrm{H}) ; \beta$-isomer: $\delta$ $1.33(3 \mathrm{H}, \mathrm{d}, J=6 \mathrm{~Hz}, 5-\mathrm{Me}), 3.08(1 \mathrm{H}, \mathrm{t}, J=9.5$ and $9.5 \mathrm{~Hz}$, $4-\mathrm{H}), 3.20(1 \mathrm{H}, \mathrm{dd}, J=3$ and $9.5 \mathrm{~Hz}, 3-\mathrm{H}), 3.24(1 \mathrm{H}$, dq, $J=6,6,6$ and $9.5 \mathrm{~Hz}, 5-\mathrm{H}), 3.53(\mathrm{OMe}), 3.64(1 \mathrm{H}$, slightly br d, $J=3 \mathrm{~Hz}, 2-\mathrm{H}), 3.67$ (3H, s, OMe), $4.65(1 \mathrm{H}, \mathrm{d}$, $J=\sim 1 \mathrm{~Hz}, 1-\mathrm{H})$.

\section{Methyl 2,3,4-tri- $O$-methyl- $\alpha$ - and $\beta$-L- rhamnopyranoside (10a and 10b)}

To a solution of 9 (96.5 mg, $0.468 \mathrm{mmol})$ in $\mathrm{MeOH}(1.5 \mathrm{ml})$ was added sulfuric acid $(30 \mu 1,0.563 \mathrm{mmol})$ and the solution was heated for 5 hours at $50^{\circ} \mathrm{C}$. TLC $(1: 1$ hexane - diethyl ether) of the solution showed two spots at Rf 0.25 (10a, major) and 0.15 (10b) (cf. 9: Rf 0.05). The solution was concentrated to a low volume, diluted with $\mathrm{CHCl}_{3}$, and washed with aq $\mathrm{NaHCO}_{3}$ and water. The organic layer was dried $\left(\mathrm{Na}_{2} \mathrm{SO}_{4}\right)$ and concentrated to give a syrupy mixture $(89.7 \mathrm{mg}, 87 \%$ ) of $\mathbf{1 0 a}$ and $\mathbf{1 0 b}$ (the ratio was $5: 1$; determined from the ${ }^{1} \mathrm{H}$ NMR spectrum). Column chromatography (1:1 hexane - diethyl ether) of the syrup 
permitted separation of the anomers.

10a: syrup, $[\alpha]_{\mathrm{D}}^{23}-59^{\circ}\left(c 1, \mathrm{CHCl}_{3}\right)$; lit. [6] $[\alpha]_{\mathrm{D}}^{24}$ $-62.5^{\circ}\left(c \quad 1.0, \mathrm{CHCl}_{3}\right) ;{ }^{13} \mathrm{C} \mathrm{NMR}\left(125.8 \mathrm{MHz}, \mathrm{CDCl}_{3}\right) \delta$ 18.2 (Me-5), 55.1 (MeO-1), 58.1 (MeO-3), 59.4 (MeO-2), 61.3 (MeO-4), 68.1 (C-5), 77.8 (C-2), 81.5 (C-3), 82.5 (C4), $98.3(\mathrm{C}-1) ; J_{\mathrm{C}-1, \mathrm{H}-1}=167 \mathrm{~Hz}$.

10b: syrup, ${ }^{13} \mathrm{C}$ NMR $\left(125.8 \mathrm{MHz}, \mathrm{CDCl}_{3}\right) \delta 18.1(\mathrm{Me}-$ 5), 57.4 (MeO-1), 57.8 (MeO-3), 61.4 (MeO-4), 62.1 (MeO-2), 72.2 (C-5), 77.5 (C-2), 82.3 (C-4), 84.4 (C-3), $102.8(\mathrm{C}-1) ; J_{\mathrm{C}-1, \mathrm{H}-1}=153 \mathrm{~Hz}$.

\section{Caprazene (11)}

A solution of $2(200 \mathrm{mg}, 0.174 \mathrm{mmol})$ in $80 \%$ aq $\mathrm{AcOH}$ $(6 \mathrm{ml})$ was heated for 2 hours at $70^{\circ} \mathrm{C}$. Concentration gave a syrup, which was added to acetone. The precipitate obtained was thoroughly washed with acetone to give $\mathbf{1 1}$ (96.3 mg, 99\%) as a colorless solid. An analytical sample was prepared by crystallization from $\mathrm{H}_{2} \mathrm{O}$-acetone, mp $210 \sim 211^{\circ} \mathrm{C}$ (dec.); $[\alpha]_{\mathrm{D}}^{19}+85^{\circ}\left(c \quad 0.5, \mathrm{H}_{2} \mathrm{O}\right)$; HR-MS (ESI, positive): $\mathrm{m} / \mathrm{z} 580.1849(\mathrm{M}+\mathrm{Na})^{+}$(calcd for $\left.\mathrm{C}_{22} \mathrm{H}_{31} \mathrm{~N}_{5} \mathrm{O}_{12} \mathrm{Na}, 580.1867\right) ;{ }^{1} \mathrm{H}$ NMR (500 MHz, $\left.\mathrm{D}_{2} \mathrm{O}\right) \delta$ $2.42\left(3 \mathrm{H}, \mathrm{s}, 5^{\prime \prime \prime}-\mathrm{NMe}\right), 2.94(1 \mathrm{H}, \mathrm{dd}, J=7$ and $12.5 \mathrm{~Hz}$, 4"'a-H), $2.99\left(3 \mathrm{H}, \mathrm{s}, 8^{\prime \prime \prime}-N \mathrm{Me}\right), 3.18(1 \mathrm{H}, \mathrm{dd}, J=5$ and $\left.14 \mathrm{~Hz}, 5^{\prime \prime} \mathrm{a}-\mathrm{H}\right), 3.34$ (1H, dd, $J=7$ and $\left.12.5 \mathrm{~Hz}, 4^{\prime \prime \prime} \mathrm{b}-\mathrm{H}\right), 3.35$ $\left(1 \mathrm{H}, \mathrm{dd}, J=4\right.$ and $\left.14 \mathrm{~Hz}, 5^{\prime \prime} \mathrm{b}-\mathrm{H}\right), 3.92\left(1 \mathrm{H}, \mathrm{d}, J=9.5 \mathrm{~Hz}, 6^{\prime \prime \prime}-\right.$ $\mathrm{H}), 4.12\left(1 \mathrm{H}, \mathrm{dd}, J=5\right.$ and $\left.\sim 8 \mathrm{~Hz}, 3^{\prime}-\mathrm{H}\right), 4.13$ (1H, slightly br d, $\left.J=\sim 5 \mathrm{~Hz}, 2^{\prime \prime}-\mathrm{H}\right), 4.20\left(1 \mathrm{H}, \mathrm{m}, 4^{\prime \prime}-\mathrm{H}\right), 4.24(1 \mathrm{H}$, slightly br d, $\left.J=\sim 8 \mathrm{~Hz}, 4^{\prime}-\mathrm{H}\right), 4.26(1 \mathrm{H}, \mathrm{dd}, J=\sim 5$ and $\sim 8$ $\left.\mathrm{Hz}, 3^{\prime \prime}-\mathrm{H}\right), 4.28\left(1 \mathrm{H}, \mathrm{dd}, J=2.5\right.$ and $\left.5 \mathrm{~Hz}, 2^{\prime}-\mathrm{H}\right), 4.34(1 \mathrm{H}$, dd, $J=2$ and $\left.9.5 \mathrm{~Hz}, 5^{\prime}-\mathrm{H}\right), 5.22\left(1 \mathrm{H}\right.$, slightly br s, $\left.1^{\prime \prime}-\mathrm{H}\right)$, $5.62\left(1 \mathrm{H}, \mathrm{d}, J=2.5 \mathrm{~Hz}, 1^{\prime}-\mathrm{H}\right), 5.82(1 \mathrm{H}, \mathrm{d}, J=8 \mathrm{~Hz}, 5-\mathrm{H})$, $6.49\left(1 \mathrm{H}, \mathrm{t}, J=7\right.$ and $\left.7 \mathrm{~Hz}, 3^{\prime \prime \prime}-\mathrm{H}\right), 7.69(1 \mathrm{H}, \mathrm{d}, J=8 \mathrm{~Hz}, 6-$ $\mathrm{H}) ;{ }^{13} \mathrm{C}$ NMR (125.8 MHz, $\left.\mathrm{D}_{2} \mathrm{O}\right) \delta 33.2\left(\mathrm{NMe}-8^{\prime \prime \prime}\right), 40.5$ (NMe-5"' and C-5"), 51.5 (C-4"'), 63.6 (broad, C-6"'), 69.4 $\left(\mathrm{C}-3^{\prime}\right), 70.7$ (C-3"), $73.9\left(\mathrm{C}-2^{\prime}\right), 75.3$ (C-2"), 77.0 (C-5'), 79.0 (C-4"), 82.7 (C-4'), 91.4(C-1'), 102.0 (C-5), 110.0 (C1"), 123.5 (C-3"'), 142.4 (C-6), 144.7 (C-2"'), 151.7 (C-2), 166.8 (C-4), $169.2\left(\mathrm{C}-1^{\prime \prime \prime}\right), 171.3$ (C-7"').

\section{(3S,3' $R)$-3-(4' -Carboxy-3'-methylbutanoyloxy)-14- methylpentadecanoic Acid (12) and (11)}

A solution of $8(240 \mathrm{mg}, 0.224 \mathrm{mmol}$ as the mono $\mathrm{CF}_{3} \mathrm{COOH}$ salt) in $80 \%$ aq AcOH $(6 \mathrm{ml})$ was heated for 2 hours at $70^{\circ} \mathrm{C}$. TLC $(2: 1: 1$ EtOAc-1-PrOH - $20 \%$ aq $\mathrm{AcOH})$ of the solution showed two spots at Rf 0.95 (12) and 0.05 (11) (cf. 8: Rf 0.35). Concentration gave a residue, which was washed with acetone to give $\mathbf{1 1}(123.5 \mathrm{mg}$, $99 \%$ ). The washings were concentrated and the resulting syrup was extracted with $\mathrm{CHCl}_{3}$. The extract was washed with water and concentrated to give $\mathbf{1 2}(82.5 \mathrm{mg}, 92 \%)$ as a colorless syrup.
12: $[\alpha]_{\mathrm{D}}^{20}+6^{\circ}\left(c \quad 1, \mathrm{CHCl}_{3}\right) ; \mathrm{MS}(\mathrm{ESI}) \mathrm{m} / \mathrm{z} 423$ $(\mathrm{M}+\mathrm{Na})^{+} ;{ }^{1} \mathrm{H} \mathrm{NMR}\left(500 \mathrm{MHz}, \mathrm{CDCl}_{3}\right) \delta 0.86(6 \mathrm{H}, \mathrm{d}$, $\left.J=6.5 \mathrm{~Hz}, 14-\mathrm{Me}_{2}\right), 1.03\left(3 \mathrm{H}, \mathrm{d}, J=6.5 \mathrm{~Hz}, 3^{\prime}-\mathrm{Me}\right), 1.15$ $\left(2 \mathrm{H}, \mathrm{m}, 13-\mathrm{H}_{2}\right), 1.23 \sim 1.32(16 \mathrm{H}, 5,6,7,8,9,10,11$ and $\left.12-\mathrm{H}_{2}\right), 1.51(1 \mathrm{H}, \mathrm{m}, 14-\mathrm{H}), 1.56(1 \mathrm{H}, \mathrm{m}, 4 \mathrm{a}-\mathrm{H}), 1.65(1 \mathrm{H}$, $\mathrm{m}, 4 \mathrm{~b}-\mathrm{H}), 2.20\left(1 \mathrm{H}, \mathrm{dd}, J=6.5\right.$ and $\left.14.5 \mathrm{~Hz}, 2^{\prime} \mathrm{a}-\mathrm{H}\right), 2.27$ $\left(1 \mathrm{H}, \mathrm{dd}, J=7\right.$ and $\left.15 \mathrm{~Hz}, 4^{\prime} \mathrm{a}-\mathrm{H}\right), \sim 2.38\left(1 \mathrm{H}, \mathrm{m}, 4^{\prime} \mathrm{b}-\mathrm{H}\right)$, $\sim 2.40\left(1 \mathrm{H}, \mathrm{m}, 2^{\prime} \mathrm{b}-\mathrm{H}\right), 2.45\left(1 \mathrm{H}, \mathrm{m}, 3^{\prime}-\mathrm{H}\right), 2.57(1 \mathrm{H}, \mathrm{dd}$, $J=9$ and $15.5 \mathrm{~Hz}, 2 \mathrm{a}-\mathrm{H}), 2.61(1 \mathrm{H}, \mathrm{dd}, J=4$ and $15.5 \mathrm{~Hz}$, 2b-H), 5.28 (1H, m, 3-H); $\left.{ }^{13} \mathrm{C} \mathrm{NMR} \mathrm{(125.8} \mathrm{MHz,} \mathrm{CDCl}_{3}\right) \delta$ 20.3 (Me-3'), $23.1\left(\mathrm{Me}_{2}-14\right), 25.5$ (C-5), 27.8 (C-12 and C3'), 28.4 (C-14), 29.7, 29.8, 29.9, 30.0, 30.1, 30.3, 34.6 (C4), 39.5 (C-2 and C-13), 40.8 (C-4'), 41.2 (C-2'), 70.9 (C3), $172.1\left(\mathrm{C}-1^{\prime}\right), 177.7(\mathrm{C}-1), 179.4\left(\mathrm{C}-5^{\prime}\right)$.

\section{$\left(3 R, 1^{\prime} S\right)-1^{\prime}-\{[N-(4-B r o m o p h e n y l)$ carbamoyl $]$ methyl $\}-$ 12'-methyltridecyl-4-[ $N$-(4-bromophenyl)carbamoyl]- 3-methylbutanoate (13)}

To a solution of $12(28.5 \mathrm{mg}, 0.0712 \mathrm{mmol})$ in THF $(1 \mathrm{ml})$ were added triethylamine ( $70 \mu 1,0.502 \mathrm{mmol})$, bis(2-oxo-3oxazolidinyl)phosphinic chloride $(40.0 \mathrm{mg}, 0.157 \mathrm{mmol})$, and 4-bromoaniline $(30.7 \mathrm{mg}, 0.178 \mathrm{mmol})$ and the mixture was stirred for 1 hour at room temperature. TLC $(3: 1$ $\mathrm{CHCl}_{3}$ - EtOAc) of the organic layer showed a major spot at $\mathrm{Rf}$ 0.55. Concentration of the resulting suspension gave a residue, which was extracted with $\mathrm{CHCl}_{3}$. The organic layer was washed with water, dried $\left(\mathrm{Na}_{2} \mathrm{SO}_{4}\right)$, and concentrated. The residue was purified by column chromatography with $\mathrm{CHCl}_{3}$ to give $13(35.1 \mathrm{mg}, 70 \%)$ as a solid. An analytical sample (prism) was prepared by crystallization from acetone, mp $160 \sim 161^{\circ} \mathrm{C} ;[\alpha]_{\mathrm{D}}^{23}-12^{\circ}\left(c 0.5, \mathrm{CHCl}_{3}\right)$; HRMS (ESI, positive): $m / z 729.1876(\mathrm{M}+\mathrm{Na})^{+}$(calcd for $\left.\mathrm{C}_{34} \mathrm{H}_{48} \mathrm{Br}_{2} \mathrm{~N}_{2} \mathrm{O}_{4} \mathrm{Na}, 729.1879\right),{ }^{1} \mathrm{H}$ NMR (500 MHz, $\mathrm{CDCl}_{3}$ ) $\delta 0.86\left(6 \mathrm{H}, \mathrm{d}, J=6.5 \mathrm{~Hz}, 12^{\prime}-\mathrm{Me}_{2}\right), 1.08(3 \mathrm{H}, \mathrm{d}, J=6.5 \mathrm{~Hz}$, $3-\mathrm{Me}), 1.15\left(2 \mathrm{H}, \mathrm{m}, 11^{\prime}-\mathrm{H}_{2}\right), 1.20 \sim 1.36\left(16 \mathrm{H}, 3^{\prime}, 4^{\prime}, 5^{\prime}\right.$, $6^{\prime}, 7^{\prime}, 8^{\prime}, 9^{\prime}$ and $\left.10^{\prime}-\mathrm{H}_{2}\right), 1.51\left(1 \mathrm{H}, \mathrm{m}, 12^{\prime}-\mathrm{H}\right), 1.60 \sim 1.75$ $\left(2 \mathrm{H}, \mathrm{m}, 2^{\prime}-\mathrm{H}_{2}\right), 2.28(1 \mathrm{H}, \mathrm{dd}, J=6.5$ and $13.5 \mathrm{~Hz}, 4 \mathrm{a}-\mathrm{H})$, $2.33 \sim 2.42\left(3 \mathrm{H}, \mathrm{m}, 2-\mathrm{H}_{2}\right.$ and $\left.4 \mathrm{~b}-\mathrm{H}\right), 2.49(1 \mathrm{H}, \mathrm{m}, 3-\mathrm{H})$, $2.55\left(1 \mathrm{H}, \mathrm{dd}, J=7\right.$ and $\left.14.5 \mathrm{~Hz}, 1^{\prime}-\mathrm{C}(\mathrm{HaHb}) \mathrm{CO}\right), 2.63(1 \mathrm{H}$, dd, $J=4$ and $\left.14.5 \mathrm{~Hz}, 1^{\prime}-\mathrm{C}(\mathrm{HaHb}) \mathrm{CO}\right), 5.25\left(1 \mathrm{H}, \mathrm{m}, 1^{\prime}-\mathrm{H}\right)$, $7.3 \sim 7.45$ (8H, m, aromatic), $7.80(1 \mathrm{H}, \mathrm{s}, 4-\mathrm{CONH}), 7.92$ $\left(1 \mathrm{H}, \mathrm{s}, 1^{\prime}-\mathrm{CH}_{2} \mathrm{CON} H\right) ;{ }^{13} \mathrm{C}$ NMR $\left(125.8 \mathrm{MHz}, \mathrm{CDCl}_{3}\right): \delta$ 21.1 (Me-3), $23.1\left(\mathrm{Me}_{2}-12^{\prime}\right), 25.8$ (C-3'), 27.8 (C-10'), 28.4 (C-12'), 29.0 (C-3), 29.7, 29.88, 29.94, 30.0, 30.1, $30.3, \quad 34.3\left(\mathrm{C}-2^{\prime}\right), \quad 39.5\left(\mathrm{C}-11^{\prime}\right), 41.1 \quad(\mathrm{C}-2), 43.1$ $\left(\mathrm{NHCOCH}_{2}-1^{\prime}\right)$, $44.2(\mathrm{C}-4), 72.0\left(\mathrm{C}-1^{\prime}\right), 117.3,117.5$, $122.0, \quad 122.1, \quad 132.3, \quad 132.4, \quad 137.2, \quad 137.3,168.7$ $\left(\mathrm{NHCOCH}_{2}-1^{\prime}\right), 170.8$ (CONH-4), 172.9 (C-1).

\section{Caprazol (14)}

To a solution of $2(150 \mathrm{mg}, 0.131 \mathrm{mmol})$ in DMF $(1.5 \mathrm{ml})$ 
was added $28 \%$ aq $\mathrm{NH}_{3}(1.5 \mathrm{ml})$, and the mixture was stirred for 4 days at room temperature. Filtration followed by concentration in vacuo gave a residue, which was thoroughly washed with acetone to give $\mathbf{1 4}(74.7 \mathrm{mg}, 99 \%)$ as a colorless solid. An analytical sample (prism) was prepared by crystallization from $\mathrm{MeOH}-\mathrm{H}_{2} \mathrm{O}$, mp $205 \sim 206^{\circ} \mathrm{C}($ dec $) ;[\alpha]_{\mathrm{D}}^{19}+28^{\circ}$ (c 0.5, DMSO); HR-MS (ESI, positive): $\mathrm{m} / \mathrm{z} 576.2155(\mathrm{M}+\mathrm{H})^{+}$(calcd for $\left.\mathrm{C}_{22} \mathrm{H}_{34} \mathrm{~N}_{5} \mathrm{O}_{13}, 576.2153\right) ;{ }^{1} \mathrm{H}$ NMR $\left(500 \mathrm{MHz}, \mathrm{D}_{2} \mathrm{O}\right) \delta 2.43$ ( $\left.3 \mathrm{H}, \mathrm{s}, 5^{\prime \prime \prime}-\mathrm{NMe}\right), 3.01$ (1H, slightly br d, $\left.J=15 \mathrm{~Hz}, 4^{\prime \prime \prime} \mathrm{a}-\mathrm{H}\right)$, $3.07\left(3 \mathrm{H}, \mathrm{s}, 8^{\prime \prime \prime}-N \mathrm{Me}\right), 3.13(1 \mathrm{H}$, slightly br d, $J=15 \mathrm{~Hz}$, $\left.4^{\prime \prime \prime} \mathrm{b}-\mathrm{H}\right), 3.20\left(1 \mathrm{H}, \mathrm{dd}, J=4\right.$ and $\left.13.5 \mathrm{~Hz}, 5{ }^{\prime \prime} \mathrm{a}-\mathrm{H}\right), 3.32(1 \mathrm{H}$, dd, $J=3.5$ and $\left.13.5 \mathrm{~Hz}, 5^{\prime \prime} \mathrm{b}-\mathrm{H}\right), 3.85\left(1 \mathrm{H}, \mathrm{d}, J=9 \mathrm{~Hz}, 6^{\prime \prime \prime}-\mathrm{H}\right)$, $4.08\left(1 \mathrm{H}, \mathrm{dd}, J=5\right.$ and $\left.8 \mathrm{~Hz}, 3^{\prime}-\mathrm{H}\right), 4.13(1 \mathrm{H}, \mathrm{d}, J=\sim 8 \mathrm{~Hz}$, $\left.4^{\prime}-\mathrm{H}\right), 4.14\left(1 \mathrm{H}, \mathrm{d}, J=\sim 3 \mathrm{~Hz}, 2^{\prime \prime}-\mathrm{H}\right), 4.20(1 \mathrm{H}, \mathrm{d}, J=\sim 5$ $\left.\mathrm{Hz}, 2^{\prime \prime \prime}-\mathrm{H}\right), \sim 4.21$ (1H, m, 4"-H), 4.25 (1H, m, 3"-H), 4.31 $\left(1 \mathrm{H}\right.$, br d, $\left.J=5 \mathrm{~Hz}, 2^{\prime}-\mathrm{H}\right), 4.39$ (1H, d, $\left.J=9 \mathrm{~Hz}, 5^{\prime}-\mathrm{H}\right), 4.44$ $(1 \mathrm{H}$, br s, 3'”-H), $5.17(1 \mathrm{H}$, slightly br s, 1"-H), $5.60(1 \mathrm{H}$, slightly br s, 1'-H), $5.82(1 \mathrm{H}, \mathrm{d}, J=8 \mathrm{~Hz}, 5-\mathrm{H}), 7.77(1 \mathrm{H}, \mathrm{d}$, $J=8 \mathrm{~Hz}, 6-\mathrm{H}) ;{ }^{13} \mathrm{C} \mathrm{NMR}\left(125.8 \mathrm{MHz}, \mathrm{D}_{2} \mathrm{O}\right) \delta 37.0(\mathrm{NMe}-$ $\left.5^{\prime \prime \prime}\right), 39.2$ (NMe-8"'), 40.2 (C-5"), $59.1\left(\mathrm{C}-4^{\prime \prime \prime}\right), 63.5$ (C-6"' $)$, 69.3 (C-3' and C-3"'), 70.0 (C-2'"), $70.6\left(\mathrm{C}-3^{\prime \prime}\right), 74.0\left(\mathrm{C}-2^{\prime}\right)$, 75.4 (C-2"), 77.6 (C-5'), $79.0\left(\mathrm{C}-4^{\prime \prime}\right), 82.4\left(\mathrm{C}-4^{\prime}\right), 91.8$ (C1'), 101.7 (C-5), 111.2 (C-1"), 142.9 (C-6), 151.8 (C-2), 167.1 (C-4), 172.7 (C-7"'), 174.1 (C-1"').

\section{Methyl 5-Azido-5-deoxy- $\alpha$ - and $\beta$-D-Ribofuranoside (16a and 16b) and Methyl 5-Azido-5-deoxy-2,3-O- isopropylidene- $\beta$-D-ribofuranoside (17)}

To a solution of $\mathbf{1 5}(860 \mathrm{mg}, 4.00 \mathrm{mmol})$ in $\mathrm{MeOH}(40 \mathrm{ml})$ was added Amberlite $\mathrm{CG} 120\left(\mathrm{H}^{+}\right.$form, $\left.2.7 \mathrm{~g}\right)$ and the mixture was refluxed for 30 minutes. TLC $(1: 2$ hexane EtOAc) of the organic layer showed spots at Rf 0.8 (17), 0.35 (16b), and 0.25 (16a) (cf. 15: Rf 0.55). Filtration followed by concentration gave a syrup, which was subjected to column chromatography $\left(10: 1 \mathrm{CHCl}_{3}\right.$ $\mathrm{MeOH})$ to give a syrupy mixture $(472.8 \mathrm{mg}, 63 \%)$ of $\mathbf{1 6 a}$ and $16 \mathbf{b}$ (the ratio was $1: 2.9$; determined by ${ }^{1} \mathrm{H}$ NMR spectrum), along with 17 (204.8 mg, 22\%) as a syrup. An analytical sample of $\mathbf{1 6 b}$ was prepared by further column chromatography $(3: 5$ hexane-EtOAc) of the anomeric mixture.

16a: ${ }^{1} \mathrm{H}$ NMR $\left(500 \mathrm{MHz}, \mathrm{CDCl}_{3}\right) \delta 2.64(1 \mathrm{H}, \mathrm{d}, J=8 \mathrm{~Hz}$, $3-\mathrm{OH}), 2.91(1 \mathrm{H}, \mathrm{d}, J=8 \mathrm{~Hz}, 2-\mathrm{OH}), 3.38(1 \mathrm{H}, \mathrm{dd}, J=5$ and $13 \mathrm{~Hz}, 5 \mathrm{a}-\mathrm{H}), 3.51(3 \mathrm{H}, \mathrm{s}, 1-\mathrm{OMe}), 3.58(1 \mathrm{H}, \mathrm{dd}, J=3.5$ and $13 \mathrm{~Hz}, 5 \mathrm{~b}-\mathrm{H}), 3.89(1 \mathrm{H}$, ddd, $J=4,6.5$ and $8 \mathrm{~Hz}, 3-\mathrm{H})$, $4.10(1 \mathrm{H}$, apparently q, $J=\sim 4, \sim 4$ and $\sim 4 \mathrm{~Hz}, 4-\mathrm{H}), 4.15$ $(1 \mathrm{H}$, ddd, $J=4.5,6.5$ and $8 \mathrm{~Hz}, 2-\mathrm{H}), 5.00(1 \mathrm{H}, \mathrm{d}, J=4.5$ $\mathrm{Hz}, 1-\mathrm{H}) ;{ }^{13} \mathrm{C}$ NMR $\left(125.8 \mathrm{MHz}, \mathrm{CDCl}_{3}\right): \delta 52.3(\mathrm{C}-5)$, 55.7 (MeO-1), 71.2 (C-2), 71.4 (C-3), 82.8 (C-4), 102.6 (C$1)$. 16b: syrup, $[\alpha]_{\mathrm{D}}^{23}-6^{\circ}\left(c 1, \mathrm{CHCl}_{3}\right)$; MS (ESI) $m / z 212$ $(\mathrm{M}+\mathrm{Na})^{+} ;{ }^{1} \mathrm{H} \mathrm{NMR}\left(500 \mathrm{MHz}, \mathrm{CDCl}_{3}\right) \delta 2.54(1 \mathrm{H}, \mathrm{d}, J=7$ $\mathrm{Hz}, 3-\mathrm{OH}), 2.70(1 \mathrm{H}, \mathrm{d}, J=4 \mathrm{~Hz}, 2-\mathrm{OH}), 3.40$ (1H, dd, $J=6$ and $13 \mathrm{~Hz}, 5 \mathrm{a}-\mathrm{H}), 3.41(3 \mathrm{H}, \mathrm{s}, 1-\mathrm{OMe}), 3.50(1 \mathrm{H}, \mathrm{dd}, J=4$ and $13 \mathrm{~Hz}, 5 \mathrm{~b}-\mathrm{H}), 4.04 \sim 4.09(2 \mathrm{H}, \mathrm{m}, 2-\mathrm{H}$ and $4-\mathrm{H}), 4.25$ $(1 \mathrm{H}, \mathrm{dt}, J=5,7$ and $7 \mathrm{~Hz}, 3-\mathrm{H}), 4.87(1 \mathrm{H}, \mathrm{s}, 1-\mathrm{H}) ;{ }^{13} \mathrm{C}$ NMR (125.8 MHz, $\left.\mathrm{CDCl}_{3}\right) \delta 53.7$ (C-5), $55.6(\mathrm{MeO}-1)$, 72.6 (C-3), 75.1 (C-2), 81.8 (C-4), 108.5 (C-1).

\section{Methyl 5-Amino-5-deoxy- $\alpha$ - and $\beta$-D-Ribofuranoside (18a and 18b)}

(a) From 16a and 16b: To a solution of the mixture (220 $\mathrm{mg}, 1.16 \mathrm{mmol})$ of $\mathbf{1 6} \mathbf{a}$ and $\mathbf{1 6} \mathbf{b}$ in THF $-\mathrm{H}_{2} \mathrm{O}(4: 1,3 \mathrm{ml})$ was added triphenylphospine $(336 \mathrm{mg}, 1.28 \mathrm{mmol})$ and the solution was kept overnight at room temperature. TLC (4:4:1 $\mathrm{CHCl}_{3}-\mathrm{MeOH}-5 \%$ aq $\mathrm{NH}_{3}$ ) of the solution showed two spots at Rf 0.35 (18b) and $0.25(\mathbf{1 8 a})$. The solution was concentrated to a low volume, diluted with water, and washed with diethyl ether. Concentration of the aqueous solution afforded a mixture (186 mg, 99\%) of 18a and $\mathbf{1 8 b}$, as a syrup. Column chromatography $(4: 4: 1$ $\mathrm{CHCl}_{3}-\mathrm{MeOH}-5 \%$ aq $\mathrm{NH}_{3}$ ) of the syrup permitted separation of the anomeric mixture.

(b) From caprazene (11): A mixture of $\mathbf{1 1}(1.23 \mathrm{~g}$, $2.21 \mathrm{mmol})$ and Amberlite $\mathrm{CG} 120\left(\mathrm{H}^{+}\right.$form, $\left.3.7 \mathrm{~g}\right)$ in $\mathrm{MeOH}(40 \mathrm{ml})$ was refluxed for 14 hours. After filtration, the resin was washed with $\mathrm{MeOH}$ and eluted with $\mathrm{MeOH}$ containing $1 \%$ aq $\mathrm{NH}_{3}$. Ninhydrin-positive fractions were collected and concentrated to afford a syrup. TLC ( $4: 7: 2: 71$ - $\mathrm{PrOH}-\mathrm{EtOAc}-\mathrm{CHCl}_{3}-28 \%$ aq $\mathrm{NH}_{3}$ ) of the syrup showed spots at Rf 0.55 (18b) and 0.45 (18a) (cf. 2: Rf 0.3). Chromatography (4:4:1 $\mathrm{CHCl}_{3}-\mathrm{MeOH}-5 \%$ aq $\mathrm{NH}_{3}$ ) over a short column gave a mixture $(184 \mathrm{mg}, 51 \%)$ of 18a and $18 \mathbf{b}$ (the ratio was $1: 2.2$; determined from the ${ }^{1} \mathrm{H}$ NMR spectrum).

18a: syrup, $[\alpha]_{\mathrm{D}}^{21}+164^{\circ}(c 0.5, \mathrm{MeOH})$; MS (ESI) $m / z$ $164(\mathrm{M}+\mathrm{H}){ }^{+} ;{ }^{1} \mathrm{H}$ NMR $\left(500 \mathrm{MHz}, \mathrm{DMSO}-d_{6}\right) \delta 1.40(2 \mathrm{H}$, br s, $\left.5-\mathrm{NH}_{2}\right), 2.58(1 \mathrm{H}, \mathrm{dd}, J=5$ and $13 \mathrm{~Hz}, 5 \mathrm{a}-\mathrm{H}), 2.65$ $(1 \mathrm{H}, \mathrm{dd}, J=5$ and $13 \mathrm{~Hz}, 5 \mathrm{~b}-\mathrm{H}), 3.28(3 \mathrm{H}, \mathrm{s}, 1-\mathrm{OMe})$, $3.70 \sim 3.76(2 \mathrm{H}, \mathrm{m}, 3-\mathrm{H}$ and $4-\mathrm{H}), 3.83(1 \mathrm{H}, \mathrm{br} \mathrm{s}, 2-\mathrm{H})$, $4.18(1 \mathrm{H}$, br s, $2-\mathrm{OH}), 4.53(1 \mathrm{H}$, br s, 3-OH $), 4.72(1 \mathrm{H}, \mathrm{d}$, $J=4.5 \mathrm{~Hz}, 1-\mathrm{H}) ;{ }^{13} \mathrm{C}$ NMR $\left(125.8 \mathrm{MHz}\right.$, DMSO- $\left.d_{6}\right) \delta 44.8$ (C-5), 55.3 (MeO-1), 70.8 (C-3), 72.3 (C-2), 86.4 (C-4), $103.6(\mathrm{C}-1)$.

18b: syrup, $[\alpha]_{\mathrm{D}}^{21}-58^{\circ}(c 1, \mathrm{MeOH})$; MS (ESI) $m / z 164$ $(\mathrm{M}+\mathrm{H}){ }^{+} ;{ }^{1} \mathrm{H}$ NMR $\left(500 \mathrm{MHz}, \mathrm{DMSO}-d_{6}\right) \delta 1.43(2 \mathrm{H}, \mathrm{br} \mathrm{s}$, $\left.5-\mathrm{NH}_{2}\right), 2.54(1 \mathrm{H}, \mathrm{dd}, J=6$ and $13 \mathrm{~Hz}, 5 \mathrm{a}-\mathrm{H}), 2.70(1 \mathrm{H}, \mathrm{dd}$, $J=4$ and $13 \mathrm{~Hz}, 5 \mathrm{~b}-\mathrm{H}), 3.22(3 \mathrm{H}, \mathrm{s}, 1-\mathrm{OMe}), 3.69 \sim 3.74$ $(2 \mathrm{H}, \mathrm{m}, 2-\mathrm{H}$ and $4-\mathrm{H}), 3.86(1 \mathrm{H}, \mathrm{dd}, J=5$ and $7 \mathrm{~Hz}, 3-\mathrm{H})$, $4.61(1 \mathrm{H}, \mathrm{s}, 1-\mathrm{H}), 4.75(1 \mathrm{H}, \mathrm{br} \mathrm{s}, \mathrm{OH}), 4.97(1 \mathrm{H}, \mathrm{br} \mathrm{s}, \mathrm{OH})$; $\left(500 \mathrm{MHz}, \mathrm{D}_{2} \mathrm{O}\right) \delta 2.69(1 \mathrm{H}, \mathrm{dd}, J=7$ and $14 \mathrm{~Hz}, 5 \mathrm{a}-\mathrm{H})$, 
$2.87(1 \mathrm{H}, \mathrm{dd}, J=4$ and $14 \mathrm{~Hz}, 5 \mathrm{~b}-\mathrm{H}), 3.36(3 \mathrm{H}, \mathrm{s}, 1-\mathrm{OMe})$, $3.92(1 \mathrm{H}, \mathrm{dt}, J=4,7$ and $7 \mathrm{~Hz}, 4-\mathrm{H}), 3.99(1 \mathrm{H}, \mathrm{d}, J=5 \mathrm{~Hz}$, 2-H), 4.09 (1H, dd, $J=5$ and $7 \mathrm{~Hz}, 3-\mathrm{H}), 4.85(1 \mathrm{H}, \mathrm{s}, 1-\mathrm{H})$; ${ }^{13} \mathrm{C}$ NMR (125.8 MHz, DMSO- $\left.d_{6}\right) \delta 45.7(\mathrm{C}-5), 55.1$ (MeO-1), 72.4 (C-3), 75.4 (C-2), 84.9 (C-4), 108.9 (C-1); ${ }^{13} \mathrm{C}$ NMR $\left(125.8 \mathrm{MHz}, \mathrm{D}_{2} \mathrm{O}\right) \delta 44.0(\mathrm{C}-5), 55.6(\mathrm{MeO}-1)$, 72.2 (C-3), 74.7 (C-2), 83.5 (C-4), 108.3 (C-1).

\section{X-Ray Crystallographic Analysis of $\mathbf{1 3}$}

A colorless prism crystal described for 13, having approximate dimensions of $0.02 \times 0.18 \times 0.40 \mathrm{~mm}$ was chosen for X-ray crystallography. The crystal data are as follows: Empirical formula; $\mathrm{C}_{34} \mathrm{H}_{48} \mathrm{~N}_{2} \mathrm{O}_{4} \mathrm{Br}_{2}$. F.W.; 708.57. Crystal system; monoclinic. Space group, $\mathrm{P} 2_{1}(\# 4)$. Lattice parameters; $a=4.883(2) \AA, b=26.383(2) \AA, c=13.875(1) \AA$, $\beta=93.970(2)^{\circ}, \quad V=1783.4(7) \AA^{3} . \quad Z$ value; 2. Dcalc; $1.319 \mathrm{~g} / \mathrm{cm}^{3} . \mu(\mathrm{CuK} \alpha) ; 31.71 \mathrm{~cm}^{-1}$. Of the 3523 reflections which were collected, 3118 were unique. The intensities of three representative reflections were measured after every 150 reflections. Over the course of data collection, the standards decreased by $6.4 \%$. A linear correction factor was applied to the data to account for this phenomenon. The structure was solved by direct method (SIR92) [16] and expanded using a Fourier technique (DIRDIF94) [17]. The non-hydrogen atoms were refined anisotropically. Hydrogen atoms were included but not refined. The final cycle of full-matrix least-squares refinement was based on 2803 observed reflections and 379 variable parameters and converged with unweighted and weighted agreement factors of $\mathrm{R}=0.089$ and $\mathrm{Rw}=0.134$. The maximum and minimum peaks on the final difference Fourier map corresponded to 0.37 and $-0.39 \mathrm{e}^{-} / \AA^{3}$, respectively. The absolute configuration of the molecule was determined based on Flack parameter, -0.076 (59).

\section{X-Ray Crystallographic Analysis of 14}

A colorless prism crystal described for 14, having approximate dimensions of $0.06 \times 0.06 \times 0.30 \mathrm{~mm}$ was chosen for X-ray crystallography. The crystal data are as follows: Empirical formula $\mathrm{C}_{22} \mathrm{H}_{33} \mathrm{~N}_{5} \mathrm{O}_{13} \cdot 4 \mathrm{H}_{2} \mathrm{O}$; F.W. 647.59; Crystal system Monoclinic; Space group $\mathrm{P}_{2}$; Lattice parameters $a=14.558(1) \AA, \quad c=11.406(2) \AA$, $\mathrm{V}=2093.4(4) \AA^{3} ; \mathrm{Z}$ value 3. Dcalc $1.541 \mathrm{~g} / \mathrm{cm}^{3} ; \mu(\mathrm{CuK} \alpha)$ $11.56 \mathrm{~cm}^{-1}$. Of the 2835 reflections which were collected, 2525 were unique. No decay correction was applied. The structure was solved by the direct method (SIR92) and expanded using Fourier techniques (DIRDIF94). The nonhydrogen atoms were refined anisotropically. Hydrogen atoms were included but not refined. The final cycle of fullmatrix least-squares refinement was based on 2525 observed reflections and 397 variable parameters and converged with unweighted and weighted agreement factors of $\mathrm{R}=0.063$ and $\mathrm{Rw}=0.099$. The maximum and minimum peaks on the final difference Fourier map corresponded to 0.27 and $-0.21 \mathrm{e}^{-} / \AA^{3}$, respectively.

Acknowledgment We thank Dr. Ryuuichi Sawa, Ms. Yumiko Kubota and Ms. Yoshiko Koyama for assistance with NMR experiment.

\section{References}

1. Igarashi $M$, Nakagawa $\mathrm{N}$, Hattori $\mathrm{S}$, Doi $\mathrm{N}$, Masuda $\mathrm{T}$, Yamazaki T, Miyake T, Naganawa H, Ishizuka M, Shomura T, Omoto S, Yano I, Hamada M, Takeuchi T. Caprazamycins A $\sim$ F, novel anti-TB antibiotics, from Streptomyces sp., 42nd Interscience Conference on Antimicrobial Agents and Chemotherapy (San Diego), Abstracts F-2031, Sept. 27-30 (2002)

2. Igarashi M, Nakagawa N, Doi N, Hattori S, Naganawa H, Hamada M. Caprazamycin B, Novel Anti-TB Antibiotics, from Streptomyces sp., J Antibiot 56: 580-583 (2003)

3. Igarashi M, Nakagawa N, Hattori S, Kinoshita N, Masuda T, Hamada M, Akamatsu Y. Caprazamycins, novel liponucleoside antibiotics, from Streptomyces sp. I. Taxonomy, production, isolation and biological properties. J Antibiot, In preparation.

4. Doi N, Igarashi M, Masuda T, Hattori S, Nakagawa N, Hamada M, Yano I, Akamatsu Y. Antimicrob Agents Chemother, In preparation.

5. Ubukata M, Kimura K, Isono K, Nelson CC, Gregson JM, Mcclosky JA. Structure elucidation of liposidomycins, a class of complex lipid nucleoside antibiotics. J Org Chem 57: 6392-6403 (1992)

6. Drautz H, Reuschenbach P, Zähner H, Rohr J, Zeeck A. Metabolic products of microorganisms. 225 Elloramycin, a new anthracycline-like antibiotic from Streptomyces olivaceus. J Antibiot 38: 1291-1301 (1985)

7. Toman R, Karácsonyi S, Palovcík R. New syntheses of mono- and di-O-methyl derivatives of methyl $\alpha$-Lrhamnopyranoside. Carbohydr Res 56: 191-194 (1977)

8. Haverkamp J, De Bie MJA, Vliegenthart JFG. ${ }^{13} \mathrm{C}$ - and ${ }^{1} \mathrm{H}-$ NMR spectroscopy of permethylated gluco-, galacto-, and manno-pyranoses and their 6-deoxy analogues. Carbohydr Res 39: 201-211 (1975)

9. Bock K, Lundt I, Pedersen C. Assignment of anomer structure to carbohydrates through geminal ${ }^{13} \mathrm{C}-\mathrm{H}$ coupling constants. Tetrahedron Lett 13: 1037-1040 (1973)

10. Ewing DF, Goethals G, Mackenzie G, martin P, Ronco G, Vanbaelinghem L, Villa P. Novel reversed cyclonucleoside analogues with a D-ribofiranose glycone. Carbohydr Res 321: 190-196 (1999)

11. Sarabia-García F, López-Herrera FJ. Studies on the synthesis of tunicamycin. The preparation of 7-deoxy-2-deamino-6hydroxy tunicamine and related products. Tetrahedron 52: 
4757-4768 (1996)

12. Mcdonald L, Barbieri L, Carter G, Lenoy E, Lotvin J, Petersen P, Siegel M, Singh G, Williamson R. Structures of the muraymycins, novel peptidoglycan biosynthesis inhibitors. J Am Chem Soc 124: 10260-10261 (2002)

13. Yamaguchi H, Sato S, Yoshida S, Takada K, Itoh M, Seto H, Otake N. Capuramycin, a new nucleoside antibiotic. Taxonomy, fermentation, isolation and characterization. J Antibiot 39: 1047-1053 (1986)

14. Muramatsu Y, Muramatsu A, Ohnuki T, Ishii M, Kizuka M, Enokita R, Tsutsumi S, Arai M, Ogawa Y, Suzuki T, Takatsu T, Inukai M. Studies on novel bacterial translocase I inhibitors, A-500359s. I. Taxonomy, fermentation, isolation, physico-chemical properties and structure elucidation of A-
500359 A, C, D and G. J Antibiot 56: 243-252 (2003)

15. Muramatsu Y, Miyakoshi S, Ogawa Y, Ohnuki T, Ishii M, Takatsu T, Inukai M. Studies on novel bacterial translocase I inhibitors, A-500359s. III. Deaminocaprolactam derivatives of capuramycin: A-500359 E, F, H; M-1 and M-2. J Antibiot 56: 259-267 (2003)

16. Altomare A, Cascarano M, Giacovazzo G, Guagliardi A, Burla MC, Polidori G, Camalli M. SIR92- a program for automatic solution of crystal structures by direct methods. J Appl Cryst 27: 435 (1994)

17. Beurskens PT, Admiraal G, Beurskens G, Bosman WP, de Gelder R, Israel R, Smits JMM. The DIRDIF-94 program system, Technical Report of the Crystallography Laboratory, University of Nijmegen, The Netherlands (1994) 